\title{
TOPONIMI RESIANI IN UNA STAMPA PER LITI DELLA FINE DEL SETTECENTO
}

\section{1.-2. Introduzione}

1. In fondo al libro di Aldo Madotto su La Val Resia ed $i$ suoi abitanti, uscito a Udine/Mariano del Friuli durante l'estate 1982, si fa cenno a una raccolta di documenti resiani stesi dal 1382 al 1784 tra Moggio, Udine e Venezia, alcuni in latino, ma nella maggior parte in italiano. Si tratta di una stampa per liti "al taglio", che il compianto amico Aldo gentilmente mi aveva dato in visione, permettendomi così di trarne: 1) un breve (as)saggio Sulla "Lingua Illirica, o sia Reseana", uscito nello stesso anno (Metodi e Ricerche, N.S., I, n. 2, Udine, 1982, pp. 94-95); 2) un esauriente elenco, fino ad oggi inedito, dei toponimi che nella raccolta di carte resiane 1382-1784 sono disseminati in grande quantità. Il fatto che alcune pagine della suddetta stampa siano già state presentate in facsimile dallo stesso Madotto (op.cit., 149-155), ci dispensa dal descriverne in questa sede l'aspetto esteriore, tuttavia riproduciamo in dimensione assai ridotta lo strano frontespizio

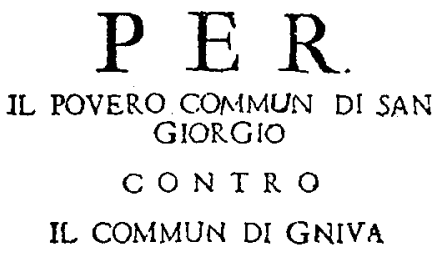

A L T A G L I O.

della raccolta di documenti riuniti in un volume unico, fatto stampare (non è specificato né dove né quando) da una delle istanze attraverso le quali dovettero passare le cause nel loro iter giudiziario. Per fortuna, la data dell'ultimo degli atti inseriti nella pubblicazione ci consente di stabilire almeno il terminus ante quem non: 13 maggio 1784.

1.1. Nei tempi andati, certe cause tra comuni resiani si protraevano per più generazioni, magari per qualche secolo; quando già sembrava che stessero per estinguersi, un ricorso imprevisto della parte avversaria le rinfocolava, sicché tornavano a divampare. Il motivo principale di queste liti era il godimento di beni comunali: boschi, "monti", pascoli, libero accesso e regresso per strada "cum animalibus". Tali contestazioni e rivendicazioni non campano di certo in aria, ma sono strettamente legate 
a località precise (loro confini, proprietà, usufrutto) e quei "beni", che hanno provocato più di una "strepitosa Litte", portano sempre un nome proprio (a volte anche due), ${ }^{1}$ in resiano.

1.2. Accanto a qualche toponimo composto c'è perfino una spiegazione in latino, friulano o italiano. Ecco per esempio che al Malevorch (1382) si accompagna un "Pizol Col" (1503); Chiamorchiaped (1503) vorrebbe dire "Sasso de Camoza" e il Masasnati Clin (1777) è reso con "Costa delli Larisi" (1503); quel corso d'acqua che i resiani tutt'oggi chiamano Bili patök (il Bieli p. dei loro vicini "Görjeni"2) ci viene servito come "Rivo Blanco" (1382), "R. Alvo" (1581) o "Rium album" (1581), mentre per Zagoslö incontriamo forme come "Drio"/ "Dietro Goslò".

1.3. Uno degli scriventi, trovatosi in questo ping-pong giuridico come giocatore e allo stesso tempo come osservatore critico, diede alla congerie di atti, tra i quali non è per nulla facile orientarsi, il nome ironico "filza di scritture" (philtia scripturarum). Orbene, da questa famosa "filza" stiamo per presentare (v. cap. 3) tutti ${ }^{3}$ i toponimi nei quali ci siamo imbattuti durante il nostro spoglio, condotto con pazienza e attenzione nel 1982. Invece di una sola forma, che potrebbe eventualmente sembrare migliore, più "giusta", più tipica delle altre, più consona alla forma che corre sulla bocca dei resiani oggi, o forse più vicina alle ipotetiche o reali forme storiche, abbiamo voluto riprodurre tutte le forme, indistintamente, anche se strampalate, ${ }^{4}$ dovute ad amanuensi superficiali (o malsicuri, perché digiuni della lingua locale), che comunque commisero evidenti errori di trascrizione, poi magari accresciuti dagli stampatori. ${ }^{5}$

1 Per il momento, purtroppo, non sappiamo ancora come spiegare questo muoversi su doppio binario: per es. "Barghin, sive/ovvero Starmaz" (1581, 1717); "Sia [Šija] ovvero Rut" (1654), "Mecia, o sia Rivo Bianco" (1777), "Puludnich, o sia Storimlin" (1749) e simili. Si tratta di semplici doppioni, cioè nomi alternativi, equivalenti, in uso per uno stesso luogo, o piuttosto di due nomi, dei quali uno è limitato solo a una parte di un sito più esteso?

2 Anche "ti görski", nome resiano per i connazionali dell'Alta Val del Torre (fiume da loro chiamato Ter, res. Tar, che nasce alle falde meridionali del Musi ed ha prestato il nome al loro parlare, "po tèrskin").

3 Volontariamente sono stati qui esclusi (perché non ci ripromettevamo alcuna "novità"!) solo i nomi dei quattro villaggi principali, già sedi dei quattro vecchi comuni: Bila /S. Giorgio (B), Njïwa /Gniva (N), Oso(j)áne /Oseacco (0), Sólbica/Stolvizza (S) e così pure la nuova sede comunale di Rávanca /Prato (R), istituita dopo l'unificazione amministrativa della valle, all'epoca napoleonica. Tuttavia, rileggere le 388 pagine del volume solo per rimediare a questa mancanza, sarebbe una fatica sproporzionata all'eventuale "utile" ricavabile.

4 A titolo di esempio ricordiamo almeno "Uva" e "Vicca" (per Učjà/Uccea), "Miruhic" e "Mirvich" (per Mirnik) e le finora non identificate località di Cescamcalle, Hirmaf, Mezanesarem, Miravolc ... (la lista con ciò non è certo esaurita). Tali nomi, oggi ancora del tutto o in parte oscuri, prima o poi potranno forse perdere la loro misteriosità ed essere identificati in base a nuove testimonianze storico-geografiche o al confronto con nomi, frutto di ulteriori ricerche toponomastiche sul campo.

5 Come errori di stampa si potrebbero riconoscere fra l'altro: Gipovaz e Gripovaz (per Lipovaz), Lastigui plas (per Lastigni p.), Sagoldi (per Sagosdi), Tamacos (per Tamaros), ecc. Non così invece 
1.3.1. Sì, anche di quest' ultima eventualità bisogna tenere conto. Ne rappresentano la prova alcune correzioni insolite, eseguite direttamente nel testo invece di essere riportate in una lista di "errata corrige" in fondo. Sorvoliamo su certi errori di datazione (oggi irrilevanti, ma allora rettificati con cura, a penna o a stampa) e scegliamo invece qualche toponimo. Sopra uno scorretto Duzzualz (p. 264) è sovrapposto (incollato!) un foglietto con su stampato Puzzualz. Ancora più caratteristica ci sembra una correzione che per maggiore evidenza riproduciamo (dalla p. 300 dell'originale):

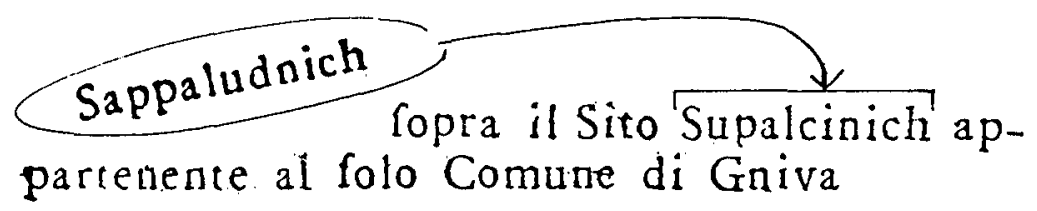

Viene da chiedersi: chi riuscirebbe a leggere correttamente la dicitura "Supalcinich", se tra le pp. 300-301 non fosse rimasto il foglietto, ormai staccato (la colla dopo 200 anni non teneva più!), con la scritta stampata Sappaludnich?

1.3.2. Tuttavia, se certe parole errate venivano sostituite, coperte con foglietti stampati aggiuntivamente in forma corretta, significa che le stampe per liti (tenendo conto che i singoli foglietti andavano ritagliati e applicati al posto giusto) dovevano avere una tiratura modesta (forse limitata a qualche dozzina di copie?). Nell'esemplare di Aldo Madotto le correzioni di questo genere non si limitano alle due or ora ricordate (Sappaludnich e Puzzualz), ma vi troviamo perlomeno altri due Puzzualz (p. 253) e un Missizza (p. 264; in questi ultimi tre casi nella fotocopia di cui disponiamo, le forme errate non si possono vedere); infine, un foglietto "vagante" tra le pp. 298-299, con la scritta stampata "Aprile" non si sa di preciso dove fosse incollato e che cosa dovesse ricoprire. Un' analisi della stampa originale e un minuzioso confronto di essa con simili pubblicazioni locali ${ }^{6}$ potrebbero forse rispondere ad alcune domande relative, per esempio, alle officine che sfornavano tali prodotti dilettanteschi, ${ }^{7}$ destinati non a una

il Porclanaz (per Potklanac), che sarà piuttosto un precoce caso di sviluppo interno nel consonantismo resiano, ossia il passaggio dalla $d$ alla $r$ : rna (da "dna"), tuw Wirne (da "Widne).

6 Vanno segnalate in primo luogo le seguenti tre: - 1) Raccolta di documenti, stampati in occasione di un processo fra i comuni di Resiutta e Oseacco, vista nel 1873 dal Baudouin de Courtenay nella chiesa di Oseacco. Esemplare incompleto, privo di frontespizio e di parecchie pagine in mezzo, monco in fondo, in $4^{\circ}, 44 \mathrm{pp}$. Riproduceva 14 documenti, i più antichi della fine del Trecento (1393, 23.3 e 22.6). -2) Nella stessa chiesa di Oseacco il BdC vide nel 1873 1' esemplare di un libro stampato "cento anni fa" (cioè intorno al 1775), in $4^{\circ}$. Mancavano le prime 42 pp., alla p. 43 cominciava un "Sommario d'investiture fatte per li magnifici signori governatori di Moggi notate per me Bernardino Andreussio, cancelliere di Moggio". Le investiture erano del periodo 1561-1639. Nell'aprile 1876 l'esemplare si trovava in mano al $\mathrm{BdC}$ e probabilmente perì a Pietroburgo nel 1918. - 3) Stampa di Pontieba, Dogna, Chiusa, Resia contro Moggio, in punto diritto di formar parte del giudizio giurisdizionale de Giurati, 1795, in $4^{\circ}$ (G. Valentinelli, Bibliografia del Friuli, Venezia 1861, p. 257); si dice che un esemplare fosse posseduto dall' avvocato Beorchia Nigris di Ampezzo.

7 Questo giudizio si riferisce solo all'aspetto esteriore, grafico, alla tecnica e non al contenuto. 
lettura normale, ma alla ripetuta consultazione da parte di giuristi "addetti ai lavori", e conservati (archiviati) dalle parti contendenti.

1.4. Abbiamo già toccato, per inciso, qualche toponimo composto (cfr. 1.2.). Si tratta di veri binomi, anche se nei documenti di solito sono resi come un'unità e non come parti separate. Potrebbe anche darsi che, nel presentarceli uniti, gli scriventi non avessero nozioni chiare sulla loro natura. Comunque, dato che l'ordine delle parole romanzo e slavo differiscono e nei toponimi resiani composti il nome sta al secondo posto, ci sentiamo in dovere di premettere al nostro elenco alfabetico del cap. 3 quanto segue.

1.4.1. Tra i circa 120 toponimi emersi dalla stampa per liti tra i comuni di Bila e Nj̈̈wa ci sono sembrati più attraenti quelli composti, non da preposizioni (del tipo Porclanaz, Tasasgarbina, Saguriziza, Sagoslò, Sagosdi, Sapoludnich), ma da aggettivi. Attorno ai 17 nomi generici identificati si sono raggruppati numerosi aggettivi con la funzione di attributi specificanti. Di volta in volta si parte dalla matrice resiana, che, se ricostruita, viene preceduta da un asterisco: per es. * gozd si ricostruisce dai casi obliqui, come ta-na Gozdë loc. sing; lo stesso dicasi di *Plaz e simili, *Brdo (invece di Bardo o Bordo), *Vrh (per Urh, Uvar, Vorch, Vorich e sim.). Sono invece senza asterisco alcuni nomi che hanno un aspetto del tutto identico non solo in resiano, ma anche nello sloveno letterario e altrove nel mondo slavo. Se disponiamo i nomi generici in ordine alfabetico, si vedranno chiaramente le rispettive frequenze; per evitare il vuoto cronologico i vari nomi sono pure datati.

* Brdo: Chilonebordo 1382; Resenebordo ${ }^{8}$ 1749; Trabnibardo 1750;

* Čelo: Chilinzolo ${ }^{9} 1382$;

* Dol/Dul: Trabnilidù 1749;

Dolina: Pradolina 1778; Prodolina 1382; Scrimizinadolina 1777;

* Gora: Monte Reisdanscaora 1717;

* Gozd: Pustigost 1605;

* Klin: Masatichen 1503; Masasnati Clin 1777;

Kolk: Malicolch, Meligolch 1777; Ravanzinculch 1609;

* Laz: Obblas 1667;

Mlin: Starimilian 1609, Starimlin 1726, Storimlin 1749;

* Peć: Belipeg 1636, Bilapech 1614, Billupeg 1723; Camorzaped 1382, Chiamorchiaped 1583; Zarnapeigh 1777; Tanavosepeg 1609;

8 Gli attributi di significato (per ora) oscuro sono sottolineati.

9 Sulla riva sinistra del torrente Črni potök, poco prima di immettersi nel Bila, c'è il colle Čelcè (dimin. di Čelö/Čalö "fronte") con alcuni maggenghi (stavoli), planïne dei Sangiorgini o "ti biski". Coloro i quali passavano l'estate con gli armenti a Čelcè erano detti Čéľ̌arji. Per l'aggettivo possessivo si può addurre un ty maš wze' no Čëlćino (tu devi sposare una di Čelcè - Matičetov 1972 $\mathrm{n}^{\circ} 20$ ) 
* Plaz: ${ }^{10}$ Bramischiblas 1622; Slonziplas 1382; Slorigi Blas 1581;

Potok: Brusinipotoc 1558, Brusignipotoch 1780; Cernipotoch 1775, Cirnipotoc 1614, Zirnipotoch 1680; Circunipotoc 1651; Forchipotoch 1614; Iacumonepotoch 1736; Crisicinipotoch 1651; Lastignipatoch 1580; Licinopetoch 1382; Nischipotoch 1499; Rischipotoch (?) 1654; Oragnipotoch 1654, Uragnipotoc 1757, Uragni potoch 1777 ; Sachipotoch 1781, Sichipoch 1636, Sighipotoch 1739, Suchipotoch 1777; Zelenipotoch 1777;

* Rava(n): Meleznarava (?) 1382; ${ }^{11}$

* Stena/Stinja: Torsca Stigna (Törska = turška stena: "Parete turca"?) 1777;

* Strnišća: Stare Stranisca 1382;

* Vrh: Malevorch 1382, Malivorich 1503; Tolstiurh 1757, Tolsti uvar 1777.

1.5. Se ci soffermiamo un momento sui toponimi semplici, il nostro occhio indubbiamente si poserà su uno dei nomi che non solo occupa uno dei primi posti nell'elenco alfabetico, ma può anche vantare una grande ricchezza di forme: è il nome

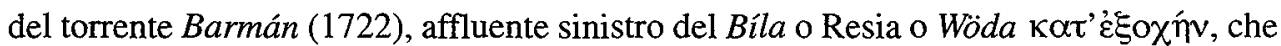
si presenta in ben 13 modi. ${ }^{12}$ Si tratta di ipertrofia cartacea, come lo dimostrano certe forme che non stanno né in cielo né in terra (Barban, Barnian, Barniant, Bartuant, Borman, Bramant, ecc.), con le quali tutti, dal primo amanuense all'ultimo compositore tipografo, sbizzarrivano la propria fantasia. Nella lista però vediamo con piacere un Barmanch (1777) che, anche se non sembra, è un fenomeno fonetico vivo (cfr. per es. benk "bene", Ц̆wank "Giovanni"), presente anche nel friulano, e produttivo, se a Bila ha figliato un گ̌wánkala (Giovannino). Nonostante la gran dovizia ci mancano però ancora due importanti forme vive e vegete senza le quali l'epifania del toponimo resiano Barman non può essere completa; avvertiamo infatti l'assenza di due tipiche forme di nasalizzazione: "ta njïvaška" tendente a $e$ come in 'Barmèn', e "ta sólbaška", vedi 'Barmón', tendente a $o$ (quest'ultima, se non si tratta di pura coincidenza, potrebbe aver lasciato un'eco parziale nella seconda sillaba di Bramon 1722). Per rimediare a simili mancanze abbiamo provveduto in due maniere: a) con il risultato dei sopralluoghi dell' amico Roberto Dapit - 1993/1994 - che si trovano al posto d'onore, cioè in testa alle singole voci, per es. come Ta-na Barménë (N); b) con citazioni principalmente dalla poesia popolare resiana, messe in nota alle singole voci.

1.6. Il nostro elenco alfabetico presenta i toponimi resiani 1382-1784 sempre al nominativo e ci lascia all'oscuro sulla loro declinazione. Dei casi obliqui ci è rimasto come unico superstite, e perdipiù problematico, un genitivo fem. della I decl.: "usque ad

10 Disponiamo anche di due traduzioni del nome geografico Plaz: Lavinam dictum (!) de Slorigi Blas (1581) e Lavina detta Slonziplas (1777).

11 Accostamento problematico, mentre per un Mezanesarem del 1382 non si può tentare nemmeno una proposta provvisoria.

12 Lo precede però Zapoludnik, con una ventina di varianti. 
summitatem Montis Cragnice" (1547, con due varianti - Cranice e Cornice - del medesimo anno, mentre il nom. Carnizza appare nel 1580). Trattamento identico hanno avuto i nomi Forchia (cum Sumitate Furche, 1614) e Njïvica /Gniviza ("Vallis parvae vocatae Gnivicae", 1547). ${ }^{13}$ La situazione però non è del tutto persuasiva: si sa infatti che la stessa desinenza, benché scritta ae, ricorre nel latino. Neanche la forma Sgarbine (1609) ci convince che si tratti di un genitivo, poiché la $e$ in questo caso si trova anche al nominativo (nasalizzazione di $a$ vicino a $n$ : come dulïne "valle", planïne "stavolo" ecc.), fenomeno particolare della fonetica di Njïwa.

1.6.1. Alla mancanza delle tipiche preposizioni resiane che collegate con qualche avverbio, fanno parte quasi integrante dei toponimi (taw/tuw, ta-čez, ta-dö, ta-gorë, ta-na, ta-nuw, ta-za, won-čez, ecc.) suppliranno almeno in parte i risultati delle recenti inchieste Dapit (1993-94). Invece, per far conoscere le forme aggettivali e qualche altro sviluppo semantico dei toponimi messi in mostra nel cap. 3, ci serviremo di informazioni provvisorie, date in nota come stralci da poesie popolari pubblicate negli ultimi trent'anni.

1.7. Qualcuno forse troverà le nostre disquisizioni troppo meticolose, ma speriamo che almeno qui (e dove poi se non in LINGUISTICA!) ci si possa permettere anche questo piccolo lusso. Se dai documenti tirati in ballo si viene a sapere che, secoli fa, giudici e altri togati, di vario rango giuridico-amministrativo, mandavano nella valle di Resia speciali commissioni, apposta per interrogare "sopra loco" vecchi pastori come testimoni, per sentire la loro opinione, degna di riguardo, sul significato di certi termini toponomastici, perché non potremmo intraprendere, "sul campo" come si suol dire oggi, qualcosa di analogo anche noi, anche se non "con protesto del giuramento in fine" (come accadeva nel passato)? Quanto riuscivano a rilevare quei "linguisti" improvvisati, poco importa se giureconsulti o pastori (per es. che "La verità fu, \& è, che la Parola Sgrasa in Lingua Illirica, o sia Reseana significa in Italiano Sfesa, o sia Schiapadura", 1777), si premuravano di farlo pervenire il più presto possibile alle istanze superiori, magari fino a Venezia, alla Corte Serenissima dei Quaranta. Se secoli fa si discuteva accanitamente per esempio sulla professata e contestata identità o diversità dei luoghi che i resiani tuttora chiamano Poludnik e Zapoludnik ("Se entro i Confini... del Sito denominato Palonich sii compreso anco il Sito detto Sapolonich", $1736 \mathrm{ecc}$.), che cosa ci impedisce, chi ci vieta di intavolare discussioni del genere, forse non del tutto "oziose", anche nel 1994?!

2. La messe di toponimi tratta dal volume a stampa rappresenta, sotto molti aspetti, un oggetto di studio molto interessante nel campo della "resianologia". Precisiamo comunque che in questa sede ci limiteremo alla presentazione del già copioso materiale, 
accompagnandolo con dati raccolti sul campo e note. Si è cercato di far luce il più possibile sui toponimi tramandati, specialmente su quelli più difficilmente intelligibili, offrendone una reale forma fonica $o$, in alcuni casi, almeno una ricostruzione. ${ }^{14} \mathrm{La}$ ricerca sul campo ha dato buoni frutti, in quanto abbiamo ottenuto il riscontro orale di 76 nomi su 117 e, a nostro avviso, ciò documenta il discreto grado di conservazione del patrimonio onomastico orale. Tuttavia, le difficoltà nella ricerca sul campo sono oggettive, poiché le abitudini degli abitanti cambiano nel tempo e, di conseguenza, il reperimento dei toponimi sta diventando un'operazione di non facile compimento. Essi si riferiscono a determinati luoghi che non vengono più frequentati perché le attività lavorative tradizionali sono state quasi del tutto abbandonate. A questo proposito bisogna tenere conto che, dal punto di vista economico, l'intera valle era caratterizzata fino ad alcuni decenni or sono da attività prevalentemente agricole, di allevamento e pastorizia. Ogni nucleo familiare possedeva del bestiame (mucche, capre, pecore) che durante l'inverno veniva tenuto in paese nelle stalle e d'estate condotto ai pascoli per l'alpeggio. Generalmente, i proprietari di bestiame possedevano anche le planïne, ossia gli stavoli dove trascorrevano i mesi estivi dedicandosi all'allevamento e alla lavorazione di prodotti caseari. La terra accanto agli insediamenti principali veniva sfruttata per la coltivazione di alcuni ortaggi come patate, fagioli, rape. Ogni altro piccolo appezzamento di terra, anche in zone molto impervie e con notevoli dislivelli, veniva invece sfruttato per la fienagione; molti luoghi dove allora si falciava sono ora ricoperti da una folta vegetazione arborea. Sappiamo quanto il fieno fosse prezioso all'epoca e quanto l'approvvigionamento spesso comportasse enormi fatiche per il trasporto dal luogo di raccolta alle stalle. ${ }^{15}$

Un'altra attività strettamente collegata al territorio era il taglio del bosco e la raccolta del legname che, dove era possibile, veniva ammassato e fatto scivolare a valle fino al più vicino torrente per la fluitazione. Sappiamo, per esempio, che da un punto del torrente Bila / Resia, nei pressi di Coritis, i tronchi venivano trasportati dalla corrente e accompagnati dai lavoranti fino al porto o preza di Ta-na Zalënin Vëro (Zamlen). Dopo essere stati recuperati, in base al segno distintivo della proprietà, ossia zlámanjë / známanjë, i tronchi venivano lì nuovamente ammassati per essere destinati alla vendita o all'utilizzo personale. Secondo le informazioni ottenute, questo era solamente uno dei vari sistemi in uso per il trasporto a valle della legna nella prima

14 Un'analisi approfondita di questo materiale onomastico sarà effettuata in un secondo momento, nel quadro dei miei studi sulla toponomastica resiana.

15 Più volte a Resia mi si racconta di un caso che sa un po' di leggendario, ossia di na učjarska, una donna di Uccea, chiamata 'Marïca Mocesa', la quale recandosi nella malga di Grubia, dietro il monte Sart, era in grado di trasportare sulle spalle una gerla (korba) piena di letame fino sul monte Kila, dove veniva scaricata per concimare un prato. La donna continuava poi la strada fino a Grubia. Dopo alcuni giorni, al momento del ritorno, la stessa gerla, probabilmente, veniva riempita di fieno e tale Marïca riprendeva il lungo cammino passando per Sart, Pučuwalca, Ronk, Püste Brdo e, dopo aver superato nuovamente il monte Kïla, scendeva a Učjà $(\mathrm{K})$. 
metà del nostro secolo e in ogni caso prima dell'avvento dei fili a sbalzo, 'net' (cfr. anche Madotto 1987, 41-50; Simonetti 1993). Tali occupazioni hanno lasciato tracce nella toponomastica, si veda per esempio il nome Ta-par ti Lï̌e (K), che dovrebbe corrispondere al frl. lisse, ossia la risina, il canalone, naturale o artificiale, che facilitava le operazioni di avvallamento dei tronchi, sfruttando, nei punti con scarsa pendenza, l'acqua delle precipitazioni nei periodi di pioggia. Un'antica testimonianza riguardante questa attività ci viene offerta pure dalla Stampa per liti del Madotto in un passo del 1499 , in cui compare un accenno ad una località dove veniva praticata, probabilmente, la fluitazione: "appresso il Rivo di Nischipotoch appresso l'Alveo del Fiume Resia, appresso un certo Conduttorio, o sia Manadore di Legni". E in ultimo aggiungiamo ancora una testimonianza dalla poesia popolare dove un inno al Črni Potök dice che in primavera questo torrente è bello e verde, ma in autunno

\section{an vináša šćándule \\ nu šcájo to womájano}

(cioè: porta fuori assi / e rami scorticati - Di Lenardo 1974, p. 40-41). Probabilmente qui spunta il ricordo della fluitazione d'una volta. Una foto documentaria e altre notizie della vecchia "chiusa" sul Rio Nero (purtroppo senza l'originario nome resiano) si trovano in A. Simonetti (1993, 78-79).

In numerosi punti della Val Resia veniva prodotta inoltre la calce nelle fornaci (furnaš) e anche di questo ci parlano numerosi toponimi del tipo Ta-na ti Ćowčïne, Ta-par Fornážo $(\mathrm{K})$. Solo nella zona di Coritis esistevano più di dieci fornaci nelle quali, spesso, si utilizzavano le pietre raccolte dalla pulizia dei prati che potevano così essere meglio sfruttati per le operazioni di fienagione.

Queste ed altre attività ancora presupponevano un legame molto stretto col territorio in cui la popolazione conduceva la propria esistenza e dove in vari modi operava. Come naturale conseguenza, gli abitanti di Resia possedevano anche una profonda conoscenza dell' ambiente e di tutte le sue caratteristiche; erano perciò in grado di individuare, attraverso un nome, ogni singolo luogo, anche molto piccolo, nella zona dove lavoravano. ${ }^{16}$ Per il resto essi conoscevano i punti di riferimento principali come

16 E' comprensibile che per un abitante di Resia vi fosse la reale necessità di mantenere vivo nella memoria il patrimonio onomastico della valle, in quanto era indispensabile per lo svolgimento delle attività di lavoro quotidiane. Il legame con l'ambiente era probabilmente così stretto che i resiani sentivano l' esigenza di dare un nome anche a piccole località. Supponiamo che vi fosse pure la coscienza che questo sapere dovesse venire trasmesso al più presto alle generazioni più giovani. Un indizio ce ne viene offerto da alcuni testi popolari, specialmente quelli narrativi, che spesso contengono numerose indicazioni di toponimi. Mi sembra indicativo il caso di una fiaba di animali della narratrice Jelica 'Tu-w Borovïčje' che contiene più di venti toponimi (cfr. Zverinice). Sembra tuttavia che una caratteristica della fiaba resiana di animali, a differenza da quelle letterarie, sia quella di descrivere avvenimenti collocati in un ambiente reale e conosciuto dall'uditorio. I bambini resiani per esempio, grazie allo spiccato realismo della fiaba, potevano venire a conoscenza gradatamente del mondo che li circondava e dei suoi svariati aspetti (Matičetov 1974-75, 113; 1973, 36). 
le montagne, i corsi d'acqua più importanti oppure determinati luoghi sulle maggiori vie di comunicazione della valle.

E' mia intenzione sottolineare il fatto che con la scomparsa delle attività economiche tradizionali, a cui ho accennato sopra, si va lentamente perdendo il contatto con l'ambiente naturale: i campi vengono lavorati in misura assai ridotta e i prati non vengono quasi più falciati; pascoli e boschi, salvo rarissime eccezioni, sono abbandonati $e$ le planïne d'un tempo stanno diventando luoghi di vacanza degli stessi abitanti di Resia. Ora, allentandosi questo rapporto con l'ambiente, anche le conoscenze che di esso avevano i Resiani si vanno perdendo, in quanto non vengono trasmesse, salvo rare eccezioni, alle generazioni successive che hanno effettuato scelte occupazionali diverse. In parte, questo patrimonio orale è ancora presente nella memoria ma è estremamente necessario effettuarne la raccolta nel più breve tempo possibile.

2.1. L'elenco dei toponimi tratti dal volume a stampa è servito come base per la ricerca sul campo degli stessi. Poiché i documenti contenuti nel libro riguardano le liti fra San Giorgio e Gniva, la ricerca è stata condotta in entrambe le frazioni presso i seguenti informatori: Maria Di Lenardo 'Marïca Č́nkina' (1928) di Bila e Severina Micelli (1914) di Podklanac per San Giorgio (B = Bila), Gino Madotto (1937) e Giuseppe Di Floriano 'Romano' (1917) per Gniva ( $\mathrm{N}=$ Njïwa). Ai dati raccolti nelle due località suddette se ne aggiungono altri, desunti dal corpus per la ricerca toponomastica commissionata dal Comune di Resia. Gli informatori che hanno fornito gli ulteriori toponimi sono: Anna Barbarino 'Maltïmpawa' (1907) di Ta-par Tigo e Silvana Paletti 'Bertulawa' (1947) di Warkota per San Giorgio, Anna Di Battista 'Fefawa' (1921) di Lischiazze (L = Lišćace), Elena Di Floriano 'Jelica Tu-w Borovičje' (1916) e Vittorio Di Lenardo 'Parïzer' (1935) di Oseacco ( $\mathrm{O}=$ Osoane), Cirilla Madotto 'Prešćina' (1925) di Coritis ( $\mathrm{K}=$ Korïto), Isidora Clemente di Uccea (1947). Le sigle tra parentesi riportate accanto ai toponimi indicano il luogo di provenienza dell'informatore.

Le fonti orali sono state riprodotte utilizzando una trascrizione fonetica semplificata e seguendo la grafia utilizzata nella maggior parte delle attuali pubblicazioni scientifiche riguardanti il resiano. A questo proposito vale forse la pena di ricordare che le cosiddette vocali scure o 'centralizzate', una delle caratteristiche principali del vocalismo resiano, vengono qui trascritte nel modo seguente: per la parlata di Gniva $\ddot{i}, \ddot{u}, \ddot{e}, \ddot{o}$, a cui si aggiungono $\partial$ e $e$ per quella di San Giorgio. Se in una parola ricorre una sola vocale scura, questa sarà sempre tonica, se invece ve ne ricorrono due, l'accento cadrà sulla prima. Nelle forme con vocali chiare invece, l'accento viene indicato normalmente, per esempio Fórćin potök.

Nei casi in cui la ricerca sul campo non abbia prodotto alcun risultato, le voci sono state ricostruite. o riportate direttamente dal libro a stampa; nell'elenco i nomi compaiono in ordine alfabetico ma accanto a Cerkwónski patök (fonte orale, trascritta secondo i criteri generalmente adottati per le lingue slave) troveremo, per esempio, un Cescamcalle (fonte scritta, con grafia di tipo italiano).

In alcuni casi, quando permane l'incertezza sulla coincidenza della fonte orale con quella scritta, il toponimo è seguito da un punto di domanda (?). I toponimi per cui è 
stato possibile effettuare la ricostruzione sono preceduti da asterisco (*). Sotto ogni voce dell'elenco, il materiale toponimico, ordinato cronologicamente, viene presentato nel modo seguente: toponimo (oppure citazione contenente toponimi), anno, giorno, mese dell'atto e pagina (tra parentesi). Alcuni toponimi sono caratterizzati da polimorfia, fenomeno frequente nelle aree con lingue in contatto. Si tratta generalmente di nomi che designano località, oppure monti e fiumi che per la loro importanza sono conosciuti anche al di fuori dell'area in questione. Alcune voci, di conseguenza, possiedono sia il nome in resiano che in italiano o friulano, ad esempio Rio Bianco Bili Potök, Resiute - Resiutta - Ta-na Bili.

Dalle fonti orali si nota che il toponimo molto spesso è formato non solo dal nome, ma anche da una preposizione o un avverbio preceduti da $t a-,{ }^{17}$ elemento che può essere seguito direttamente da una preposizione ( $n a$, par, pod, $w$, ecc.) oppure da un avverbio come gorë / hurë / yorë / orë 'sù' (a seconda della località) più la preposizione; generalmente ciò comporta anche l'uso di un caso obliquo come il locativo, per esempio con ta-gorë $w$, o dell'accusativo con $t a-z$; ta- si fonde anche con un altro

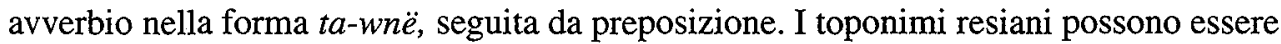
preceduti anche da avverbi assieme a preposizione ma senza l'elemento ta-: per esempio dö par, gorë $z$, un/won/wun na. Osservando invece i nomi tratti dal volume, ci rendiamo conto che sono stati tramandati quasi esclusivamente senza ta- e spesso anche senza preposizione, per esempio Porclanaz Ta-pot Klancon, Tamoro Ta-par Tamoru; rarissimi casi con ta-: Tasasgarbina $\sim$ Ta-za Škarbino, Tanavosepeg. ${ }^{18}$

Come già accennato sopra, la ricerca sul campo in alcuni casi rimane senza risposta. Per determinate forme tramandate si può allora ricorrere al confronto con altri documenti che riproducono $\mathrm{i}$ toponimi in forma più chiara e fedele alla pronuncia dell'epoca. In una pubblicazione del 1967, Resia. Numero unico, vengono per esempio riportati i toponimi da una carta datata 9 giugno 1672 ed ecco che un Scrimizinadolina contenuto nel nostro corpus perde forse la sua misteriosità se lo si accosta a Smirizina Dolina (prob. "Valle degli abeti") della carta antica. Anche le opere di Baudouin de Courtenay, che in genere ha effettuato una fedele trascrizione del materiale (ricco di toponimi) rilevato sul campo, ci possono a volte essere d'aiuto. Tuttavia, dal momento che in questo contributo non ci occupiamo di etimologia, se non occasionalmente, ci riserviamo di trattare le eventuali analisi di tipo storico-linguistico in un'altra sede.

17 Tale aspetto morfologico caratterizza in particolare le preposizioni e gli avverbi, e perciò anche i toponimi, non solo della Val Resia ma anche delle Valli del Torre. Per le Valli del Natisone non abbiamo elementi sufficienti per esprimerci con sicurezza, ma vi si può notare un uso sporadico di queste forme.

18 In certi casi, per non staccare eccessivamente la forma scritta da quella orale, abbiamo indicato nell'elenco la sola base, a volte ricostruita, del toponimo, seguito dalla testimonianza raccolta sul campo. Cfr. per esempio Oblaze: Ta-dö z Obláze (K), Wobláze (B). 


\section{Elenco dei toponimi dalla stampa per liti 1382-1784}

\section{Bargïn (B), Barhï (N) ${ }^{19}$}

- Pascum unum Montis Mussi siti in Jurisdictione dicti Castri Tarcenti nominatum Barghin, sive Starmaz. 1581.29.5 (25)

- conferma sul formaggio pagato come affitto per l'alpeggio su 'la Monte di Bargona'. 1617 (334)

- le Montagne di Mussi, e Bargona... 1716.21.9 (335)

- le Montagne di Mussi, e Bargogna. 1717.26.8 (335)

- un Pasco della Monte di Musi chiamato Barghin, ovvero Starmaz. 1717.2 .9 (57)

- Item il Pascolo del Monte Musi, situato nella Giurisdizione del Castello di Tarcento nominato Barghin, sive Stormaz. 1778.12.3 (220)

- Pascolo del Monte Musi (Tercento) nominato Barghin, sive Stermaz. 1781.6.3 (279)

- il monte Musi nominato Barghin, sive Stermas.1783.9.7 (312)

\section{Barmán: Ta-na Barmáne (B), Ta-na Barménë (N)}

- Barban: Rivo Barban. 1777.29.4 (211)

- Barman: legnami tagliati nei siti promiscui di Sappolodvich e Barman. 1722.24.1 (80), Barman. 1722.8.10 (86), 1723.9 .11 (97), 1724.1 .4 (100, 102), 1736.13.10 (340), 1736.1.12 (342), 1777.12.3 (220)

- Barmanch: Rivo Barmanch. 1777.29.4 (212)

- Barmand: Rivo denominato Barmand. 1723.9.11 (95)

- Barmant: 1722.8 .10 (86), 1723.9 .11 (96), Strada di Barmant. 1723.9.11 (98), Barmant. 1736.17.11 (341), 1779.22 .6 (224), 1780.4 .9 (263, 264, 265), 1781.13.2 (299), 1781.6.3 (277), 1781.9.7 (311), Comunale Barmant. 1783.21.6 (367-8)

- Barnian: Rivo Barnian. 1777. 29.4 (211)

- Bartuant: 1723.4.11 (93)

- Berman: 1777.25.2 (214)

- Bermant, o sia Bramon: $1722.22 .3(84)$

- Borman: 1779.22.6 (226, 227)

- Braman: nelli siti e Territorio di Braman, e Sapoloduvich promiscui tra loro Communi sino al confine di Misizza. 1721.21.11 (79), Braman. 1722.7.3 (82), 1722.17.10(90)

19 Su di un foglio volante stampato a Gemona nel 1930 sta erroneamente scritto "róse te branünave", che in Ce fastu? n $\mathrm{n}^{\circ}$ 1-43 (Udine, 1965-67, p. 454, nota 5) già venne corretto in Brahýnawe, ma qui si rettifica ancora una volta: Brahýnave (potrebbe essere anche Barhýnave). E' uno dei nomi alternativi per la stella alpina (Leontopodium alpinum) che cresce anche sul Musi, però più in alto del pascolo o "monte" Bargin che ha prestato loro il nome. 
- Bramant: 1724.27.4 (104), 1724.17 .6 (106), 1725.13.1 (112), 1725.16 .3 (113), 1725.7.5 (117)

- Bramon: $1722.22 .3(84)$

3. Bîla $(\mathbf{K})^{20}$

- Flumen Bible. 1382.9.7 (3) (cfr. Lazones)

4. Bíla pëć $(\mathbf{N})$

- Confinante ab Orta cum Rivo de Bilapech. 1614.16.8 (36)

- Bona Comunalia in Monte dicto Belipeg in Parte umbrae in illis tamen locis, in quibus possint reduci in medilibus mediante destrutione nemoris. Item bona Comunalia post Montem Belipeg prędictum in facię Solis in loco vocato Polunich... 1636.11 .7 (337)

- ...attraversando sotto Planiniza s'estendono sino al Luoco detto Billipeg, e Cirnipotoch, che è a Ponente. 1723.9.11 (95)

- Billipeg. 1723.9.11 (97)

- nella Monte di Billapeg. 1753.1.9 (182)

- il sito Comugna detta Monte Bilapeg. 1753.1 .9 (183)

- nel Monte di Bilapech. 1756.27.2 (124)

- alla sommità di Bilipeg. 1777.29.4 (212)

- una parte delli Monti Billapeg. 1780.10.6 (254)

- Monte Belipeh. 1780.10.6 (254)

- Monte Bellipeg. 1780.4.9 (265-266)

- Bilapeg. 1782.9.7 (312)

\section{Bramischiblas}

- il Tamoro chiamato del Pozzo, ovvero Bramischiblas. 1622.2.8 (78)

6. Brëzavice (B, N)

- Bresaviza. 1749.15.5 (51)

- Bresaviza. 1778.12.3 (219)

20 Nella valle l'unico corso d'acqua meritevole della qualifica "flumen" è il Bila. Se nella stampa per liti del Madotto si parla anche dello "Alveo del Fiume Resia" (1449), un'infinità di recenti testimonianze letterarie (orali e scritte) parlano in favore di Bila. Così, per es., in un canto registrato a Korïto nel 1963, a un giovane collerico è rivolto un consiglio: Da nutar $h$ Bili na pujdè / nu trykrat wodo prabradè, / ka jiti wum će ji prejtèt (Matičetov $1972 n^{\circ} 40$ ); cioè: Se ne vada giù al Bila, / guadi tre volte l'acqua, / che la rabbia le passerà. Precisiamo che le traduzioni dei versi tratti dai canti resiani date tra virgolette sono da intendere come citazioni autentiche (ad notam et litteram) mentre le altre sono solo approssimative, riassuntive e correttive. 


\section{Brüsin patök $(\mathbf{B})^{21}$}

- Brusinipotoc. 1558.31.5 (30)

- Brusinipotoch. 1558.31 .5 (31)

- sino alla Bassura di Brusinipotoch. 1654.5 .10 (167)

- Ad instanza del Commun di San Giorgio.... comettemo a miss. Zuanne Saurano di Campolar, che... non debba nè per se, nè per terze Persone tagliar, nè far tagliar alcuna sorte d'Arbori nel Bosco chiamato Brusinipotoch, in Curnich Sagoslò, nè li tagliati, da lui ammovere... 1667.12.10 (40)

- appresso Rivo Brusinipotoch. 1746.29.4 (49)

- con il Riuselo drio il stali di Domenico Barborino, che discende al Rivo Sagata detto Brusinipotoch. 1749.3.3 (50)

- Brusinipatoch. 1749.15.5 (52)

- Brusinipatoc. 1749.15.5 (52)

- ...Peragiazzà, indi suso per la sommità del Monte, indi calandosi in Drusini Potoch drio Goslò, indi suso in Clivisghie e Jamnich, indi suso alla sommità Tolsti Uvar ponte ombra, calandosi per la Monte, in Rivo Dusinich, indi seguitando detto Rivo sino al Fiume Resia, similmente seguitando detto Fiume sino al detto Rivo Uragnipotoch. 1777.29.4 (210)

- Brusinipotoch. 1780.11.3 (238, 239)

- Brusignipotoch. 1780.11.3 (241, 243)

- Brusinipotoch. 1780.18.4 (246, 247)

\section{Cammin}

- a Meridie Cammin, Montis qui est supra Montem nuncupatum Monte Maggior. 1581.6.6(27)

\section{Casaria}

- e nel Monte Casaria. 1779.22.6(224)

- Monte Casaria. 1779.22.6 (227)

21 Se dopo due secoli da quando ininterrottamente si usarono forme del tipo Brusinipotoc, con lievi differenze solo nella seconda parte del composto (Patoc, Potoch: 1558, 1654, 1667, 1746, 1749), nel 1780 accanto alla stessa forma ne compare una "nuova" (?), palatalizzata: Brusignipotoch; questo ci ricorda l'uso, nel Seicento promiscuo (?), di Branizza o Brenizza/Bregnizza, affluente sinistro del frume Vipava (Liber Baptizatorum I, Tomaj, archivio parrocchiale). Per quel torrente, ancora oggi, si sentono alternativamente due nomi: Breníca e Brenjíca; potrebbe darsi quindi che anche il Brusignipotoch resiano non sia un semplice "errore di stampa". Ma in questo momento conosciamo soltanto forme non palatalizzate, tra le quali è degna di ricordo quella che sì trova in una poesia inedita (ma dall'autrice, Silvana Paletti, già varie volte recitata in pubblico, anche alla radio), che ci guida delicatamente colà, dove sgorga... tu ke wzíra / wöda mâ ta Brüsinawa (informazione avuta per telefono). 
- Il povero Commun di S. Giorgio...ha sempre pacificamente goduto li Pascoli del Monte Casaria nelli siti di Ucea fino al Livinal Mirnich... 1779.15 .9 (229)

- ...Pascoli esistenti nel Monte Casaria nelli Siti di Ucea fino al Livinal di Mirnic. $1779.15 .9(230)$

\section{Cerkwónski patök (B), Réjnek potök (O)}

- Circunipotoc. 1651.6 .5 (161) (cfr. sub Križačin patök)

\section{Cescamcalle}

- Cescamcalle. 1382.9.7 (3) (cfr. Meleznarava)

\section{Colasnich}

- Item un altra Pezza in Colasnich. 1609.16.8 (32)

- un altro pezzo in Colasnich appresso il pascolo Commune. 1726.9.4 (48)

- Colosnich. 1778.12.3 (218)

\section{Colluto}

- Scrassa: Noi poi lo [cioè quel sito, Scrassa] chiamiamo il Costone, che viene a dar termine in altro sito chiamato il Colluto, \& indi poi passa alla strada, che conduce retamente al luoco chiamato la Forca nel sito poi Capitolato, ho sentito qualche volta da Pastori stessi chiamar Torska Stigna. 1777.27.5 (364)

\section{4. Čárna pëć (N), Č́rna pëć (B)}

- item bona Comunalia post Montem Belipeg prêdictum in facie Solis in loco vocato Polunich juxta ab ortu Rium Sichipotoch, a Meridie dictum, ab Ocasu locum de Cernipeg, \& a Septentrione locum vocatum Cufer (!). 1636.11.7 (337)

- suso per la sommità di Cuzer sino al Zarnapeigh, caminando poi alla Bassura di Forchia... 1777.29.4 (211)

\section{5. Čárni patök (N), Čárni patök $(B)^{22}$}

- nel Monte Discarbina (!) verso Rivo de Cernipotoch. 1605.9.1 (75) (rinnovazion Bando 1597)

22 Di questo bel nome, che però incute anche paura, si fregiano: a) un affluente di sinistra del Bila, il vero e proprio Č.P., noto per le rovinose piene autunnali che travolgono quanto si oppone alla loro furia e che fino al 1837 (anno di trasferimento sulla destra del Bila dell'unica strada carrabile per Resia) era una perenne minaccia per il libero accesso alla parte abitata superiore della valle; $b$ ) l'omonima, non meno famosa göra ("la monte", pascolo comune, di proprietà del paese di Bila, situata vicino alle sorgenti del Č.P.); le relative costruzioni di ricovero per pastori, bestiame e attività casearia sono però ormai da tempo in rovina; c) borgatella di 2-3 case, poco distante dallo sbocco del Č.P. nel Bila, già insediamento stabile di alcune famiglie provenienti da Bila, ma abbandonata e trasformata in sadïn ("sedimen", se usiamo il termine che si trova nella stampa per liti del Madotto); d) la nota danza "ta černipatókawa", cavallo di battaglia dei danzatori resiani che la alternano al canto "Lipi moi Cerni Potoc" (Di Lenardo ${ }^{\circ} 17$ ). 
- Rivo de Cirnipotoch. 1605.9.1 (75)

- de nemore Scarbina in Rivo de Cirnipotoch. 1613.24.6 (34)

- Bosco nel Monte di Cirnipotoch. 1667 (45)

- nella Monte di Zirnipotoch. 1680.28.3 (44)

- Cima di Cirnipotch. 1723.9.9 (95)

- Cirnipotoch Montagne di ragione di San Giorgio. 1723.9 .9 (96)

- Cernipotoch. 1723.9 .9 (98)

- nel Monte di Cirnipotoc. 1749.15 .5 (51)

- le pendenze di Cirnipotoch. 1753.25.2 (53)

- Cernipotoch. 1775.27.7 (356)

- Andrea Nais Conduttor di detto Monte Cirnipotoch. $1775.27 .7(356)^{23}$

- Monte Cirnipotoch. 1775.27.7 (357)

- Cernipotoch. 1776.26.6 (360), 1777.27 .5 (365)

- Pascoli, Boschi, e Montagna di Cirnipotoch. 1777.12.3 (219)

- Cirnipotoch. 1777.27.5 (364)

- Montagne Cernipotoch. 1780.10.6 (254)

- Cernipotoch. 1780.4.9(265)

- Cirnipotoch. 1781.6.3 (278), 1782.9 .7 (311)

16. Ćadïn $(B, N)$

- Caminando via per Cadin, Lavori...1605.9.1 (75) (rinnovazion Bando 1597)

- drio Musi, di poi a dritta Linea sino in Campo di Cadin, indi caminando suso per la sommità di Stante, discendendo in Scrasa di Planneniza, indi alla Bassura di Forchia, indi discendendo, e caminando per il Rivo Suchipotoch sino in altro Rivo Barnian $1777.29 .4(211)$

- Cadin. 1777.25.2 (214)

- La verità fù, \& è, che il Trozzo per il quale si và in Cadin si chiama Sgrasa di Planiniza... 1777.7.6(362)

- Cadin in Zorgnale. 1778.12.3 (219)

17. Ćamúrčina pëć $(\mathbf{N})^{24}$

- Camorzaped. 1382.9 .7 (3) (cfr. Chilonebordo)

- ...a loco, qui dicitur Chiamorchiaped, quod latine interpretatur, Sasso de Camoza...1503.5.7 (7)

23. Nella Val Trenta il 'conduttor' viene chiamato spravnik, per la Resia invece non conosciamo il termine originale resiano per la persona che stava a capo della "monte" (göra), però il responsabile delle latterie in paese era chiamato fadár che trova un riscontro nel frl. fedâr, cfr. fede 'pecora'.

24 Cfr. Matičetov 1982. 


\section{Darnúwa (B)(?)}

- Dornove. 1749.15.5(51), 1753.25.5 (53)

\section{Dusinich}

- in Rivo Dusinich.1777.29.4 (210)

\section{Forcastina}

- discendendo poi in Scrassa di Planinizza, o sia Forcastina. 1778.12.3 (219) (Cfr. Tölska stina)

\section{Fórća (B, N)}

- confinante...a Meridie cum Sumitate Furche. 1614.16.8 (36)

- indi alla Bassura di Forchia. 1777.29.4 (211)

- Bassura di Forchia. 1777.29.4 (212)

- la Forca. 1777.27.5 $(364,365)$

\section{Fórćin patök (B), Fórćin potök $(\mathrm{N})$}

- Confinante...ab Occasu cum Rivo de Forchinipotoch. 1614.16.8 (36)

23. Golìc (B), Holác (N) ${ }^{25}$

- Goliz. 1605.9.1 (75), 1778.12.3 (219)

- Galiz. 1749.15.5(51)

24. Goslö (B) ${ }^{26}$

- Goslò. 1651.6.5 (161)

25 In un canto di Bila (Di Lenardo $1974 \mathrm{n}^{\circ} 49$ ) sono ricordate due coppie di montagne opposte, lontano all'orizzonte, corrispondenti più o meno ai 4 punti cardinali: Morjana (Ovest) nu Ćanen (Est), Pućimuni (Nord) nu Golic (Sud). L'Amariana e il Pisimoni sono al di là del Fella, si ergono nel territorio friulano, il Canin e il Goliz sono invece "di casa", resiani. Né in questo né in altri canti resiani simili si tratta però di semplici menzioni geografiche, ma vi si nascondono dei significati metaforici. Particolarità resiana, con una primordiale carica poetica: sia a sfondo storico, per es. Morjána nu Ćanèn / te ninki nur vaćüwalo... (L'Amariana [= i friulani] e il Canin [= i resiani] in un lontano passato avevano avuto delle cause giudiziarie, "vece", per il possesso di pascoli - Matičetov 1978, 162 ssg.), sia d'ispirazione puramente poetica, come gioco di fantasia: Da gora ta Ćanynina / nu gora ta Banërina / pojütra ko ni wstaneta / ni sa saludawata... (Il monte Canin e il monte Banera, al mattino quando si alzano, gentilmente si salutano... - Matičetov, $1972 \mathrm{n}^{\circ}$ 10). A proposito dell'agg. poss. Ćanynina: questa inattesa desinenza femm. sarà forse da attribuire all'analogia con Banërina oppure a un forte influsso di göra; normalmente avremmo Ćanynawa/Ćaninawa/ Ćaninova, come si vede in parecchi canti (per es. Matičetov $1972 n^{\circ} 9$ e Kumer $1975 \mathrm{n}^{\circ} 35,36,306$ ). Tuttavia conosciamo anche altri "strappi alla regola": za gor 6 Gumynino o buraška Pustiözdina, dove le rispettive basi maschili - Gumin e Pustiözd richiederebbero una formazione aggettivale differente: Gumynawa e Pustiözdawa.

26 Nel nostro elenco Goslò è l'unico toponimo sempre munito di accento. Dell'importanza di questo "sito" ci parla, accanto a una ricca serie di testimonianze, a partire dal ' 500 , anche il fatto che 
- Goslò di Gniva. 1654.5 .9 (167)

- Caslò.1659.19.1 (170)

- Crisizis, Goslò e Curnich. 1756.27.2 (124)

- Goslò. 1757.21.12 (142)

- Un [Bene] Communale detto Goslò, sotto Goslò, e drio Goslò goduto in promiscuità col Commune di San Zorzi. 1777.29.4 (210)

- in Monte noncupato Goslò.1778.15.12 (280)

- Goslò.1778.15.12 (281, 282)

- ...tagliar Legne...da una, e l'altra parte del Monte Goslò...1780.11.3 (237)

- Goslò. 1780.10.6 (251)

- Monte Goslò. 1780.10.12 (289, 290)

25. Göst (B), Ta-na Huzdë (L) ${ }^{27}$

- ...un altro pezzetto Communale sotto li Prati di Gost, confina a Sol levante con il Rivo Barban, a Mezzogiorno colli Prati di Post (!), Sol a Monte con li Prati di Las, Settentrion col Fiume Resia, indi caminando contro il Fiume sino al Rivo detto Potoch verso Levante. 1777.29.4 (211)

\section{Grachicinich}

- suso alla sommità di Grachicinich (!), ove similmente si trova altra Croce...1777.29.4 (212)

\section{Hirmaf}

- Rium dictum di Hirmaf. 1581.6.6. (27)

\section{8. *Hliviǐšce: Klivišśé, Tu-wnë na Kliviššé (O)}

- verso Curnich, Hlivise, e drio Goslò. 1746.29.5 (49)

- indi suso in Clivisghie. 1777.29.4 (210)

\section{Jacumonepotoch}

- un suo pezzo di Medilli nel Monte Sapaludnich chiamato Jacumonepotoch. 1736.13.10 (339)

\section{0. *Jamnik, Jáwnik (B) ${ }^{28}$}

- ...Clivisghie, e Jamnich, indi...Tolsti Uvar...1777.29.4 (210) (cfr. Brüsin patök)

questa zona ha trasmesso il nome a un gelido vento resiano che spira da NE, il "goslár": ...pleše dem I na muziko wod goslarja, / ke naganja snih (dalla poesia To balanà /Nevica di Silvana Paletti, in Sodobnost, 28, 1980, p. 1141), ossia: "Il vento danza / alla musica del goslár, / che mena la neve" (cfr. La Battana, 18, ${ }^{\circ}$ 63-64, Fiume, Marzo 1982, pp. 122-123).

27 Per via del Barmán in piena ta na Gözd na moren tet (su a G. non posso andare: Matičetov $1972 \mathrm{n}^{\circ}$ 51). 


\section{Karnïca (B), Ta-na Karnïci (N) ${ }^{29}$}

- ...super loco Montis Cranize. 1547.25.6 (9)

- Confinis....Montis Cornice. 1547.25.6(10)

- usque ad summitatem dicti Montis Cragnice. 1547.25.6 (11)

- Montis Cranice. 1547.25.6 (11)

- ad dictum Montem de Cornica. 1547.2.7 (12)

- Intervenientes vero pro Communi, \& Hominibus Gnivae elegerunt, acceptaverunt, \& approbaverunt in partem \& portionem suam Montem vocatum de Carnizza. $1580.21 .6(16)$

- li pascoli delle loro Montagne di Casaria, nominate di Carnizza. 1637.19.12 (19)

- Carnizza. 1723.9.11 (96)

- Carniza. 1723.9.11 (97)

32. Kila $(\mathbf{B}, \mathbf{N})^{30}$

- Montem qui vocatur Chilla. 1382.9.7 (2) [monte nominato assieme a Prodolina e Stare Stranisca (!): omnes Montes contiguos et ad invicem se tenentes]

\section{Killine bárdo (O)}

- versus partes orientales est quidam locus vocatus Chilonebordo usque in Chilinzolo, \& in Camorzaped. 1382.9.7 (3)

34. *Kiline čalö ${ }^{31}$

- Chilinzolo. 1382.9.7 (3)

28 L'informatore (B) conosce una località Jáwnik nella zona di Tulsti war, tuttavia in Baudouin de Courtenay (1876: 335) ritroviamo il toponimo tòw Jámnice, che corrisponderebbe al loc. di *Jamnik.

29 Di Karnüca esiste un dimin. Karnüčica, con valore affettivo: [S. Anna] na uarie usò Carnicizo ("custodisce tutta C." - Di Lenardo $1974 \mathrm{n}^{\circ}$ 51). Però il diminutivo Karnï̌ica ha anche il significato di "canto sulla Madonna di Carnizza" (v. Matičetov 1964, 128 ssg.). Agg. poss.: jöra ma Karnycina (alla lettera sarebbe, certo, "il monte di K.", però nella traduzione in sloveno letterario si è ricostruito, secondo il senso, l'originario wöda "acqua" (Matičetov $1972 \mathrm{n}^{\circ} 51$ ).

30 Da ta čiz Kïlo, ta čiz Kaw / stojï koraski den Učjár (Dalla Chila al Cal / sta a gambe divaricate un uomo di U.). L'agg. poss. si forma normalmente: or cez to öro Kilino (su oltre il monte Ch. Matičetov $1972 \mathrm{n}^{\circ}$ 35); Da göra ma ta Kylina (Quel mio monte di Ch. - Merkù $1976 \mathrm{n}^{\circ} 521$ ).

31 Per la ricostruzione del secondo elemento di Chilinzolo in Čalö ci siamo avvalsi oltre che del dim. Čalcë (v. nota 9) del nome di una località lungo la strada che porta a Rüšce-Ruschis, villaggio estivo dei pastori di Bila: Ta pod Cialon te bili uch, / ta na Cialè so vedamzi... "Sotto Cialò c'è un lupo bianco, sopra C. i fuochi fatui" (Di Lenardo $1974 n^{\circ} 11$ ). Da non dimenticare che quelli di Bila "ti biski" possedevano qualche pascolo anche dalle parti di Kila. 
35. Krï̌race $(B)^{32}$

- ...li staulieri di Crisizza e Goslò. 1651.6.5 (161)

- Crisazza. 1651.6.5 (161)

- Crisizis. 1659.19.1 (170)

- ...nei Boschi posti nel loco chiamato Crisizis, Goslò e Curnich... 1756.27.2 (124)

- Crisizzis. 1757.26.10 (139)

- Crisazis. 1757.21.12 (142, 143)

- Crissis. 1759.18.4 (153)

\section{Krïžacin patök (B)}

- ...li staulieri di Crisizza, e Goslò confinanti da Levante Rivo di Crisizinipotoc, Meridie Fiume Resia, Ponente Rivo di Uragnipotoch, e Circunipotoc, e Settentrione la Somità di Curnich... 1651.6.5 (161)

- Rivo chiamato Crisicinipotoc. 1654.5 .10 (167)

37. Kücer $(B, N)^{33}$

- Caminando in cima la Monte Scarbina passando via per Cuzer, \& tutto Cernipotoch suso, per la Scrassa di Planinizza, \& Stanssissa caminando via per Cadin... 1605.9.1 (75) (rinnovazion Bando 1597)

- a septentrione locum vocatum Cufer (!). 1636.11 .7 (337)

32 Come corollario su quanto detto in nota 35, a Lïpovac, rispettivamente a Ravanca, il Trankon localizzava un "paradiso" e un "inferno" resiani, il purgatorio invece si troverebbe a Križaca: purhatori ta na Crisaze (Di Lenardo $1974 \mathrm{n}^{\circ} 23$ ). Come spiegare questo enigma? Che ci possano essere di mezzo la pubblica moralità, ce lo fa supporre un altro canto, dettato a (J)ama nel 1964

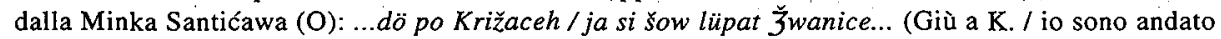
a bussare alla G. - Matičetov $1972 n^{\circ} 32$ ); la Giovannina di K. generosamente invitò il visitatore ad entrare nel proprio letto per "scaldarsi un poco". Solo in base a una documentazione più ricca si potrà stabilire se il nome della località sia un neutro sing. o un plur. tantum.

33 Si può effettuare una distinzione precisa tra Kücer (Cuzzer) e Kucëra ? Il secondo nome non compare nei documenti 1382-1784, tuttavia non osiamo definirlo frutto della poesia "toponimica" resiana degli ultimi duecento anni. Stando all'ultima informazione, Kücer sarebbe ta vlika glawa wod Škarbïne; sa vïdi nuw Banitke (A.P., Warkota, 10.11.94). Una chiara testimonianza del toponimo appare nel seguente passo: To bila na Culáuaua, / chi na Cuzer na ie slà "Era uno dei Culau/ che è salito sul Cuzer..." (Di Lenardo $1974 \mathrm{n}^{\circ} 44$ ). L'originale parla di una Kulawawa, salita sul Kücer, ma per ragioni prosodiche, per avere dei regolari ottonari, il canto resiano sottintende una "rožica", amato o amata, "lui" o "lei"; soltanto da informazioni aggiuntive avute lì per lì dall'informatore, si riesce a conoscere la realtà... Ecco ancora uno dei tipici canti resiani dove il Baudouin de Courtenay si lamentava che, pur essendogli tutto chiaro filologicamente, non riusciva ad afferrare il significato dell'insieme. Altre testimonianze parlano piuttosto in favore di Kucëra: Ta na Cuzëri to harmi "Sul monte Cuzera sta tuonando..." (Di Lenardo $1974 \mathrm{n}^{\circ} 15$ ); una (problematica) höra ta Kucörine ci è data da Z. Kumer (19.75 n 305); Ta-za Kucëro to garmy "Dietro il monte Cuzzer [!] tuona" (Merkù $1976 n^{\circ} 525$ ). Ma in quest'ultimo caso crediamo che si tratti senz'altro di Kucëra e non di Kücer. 
- in Scarbina per Cuzer per Resenebordò. 1749.15.5 (51)

- per la Sommità di Cuzer sino al Zarnapeigh. 1777.29.4 (212)

- Cuzzer. 1778.12.3 (219)

\section{Kürnik (B)}

- nel bosco chiamato Brusinipotoch, in Curnich Sagoslò... 1667.12.10 (40)

- Curnich. 1680.28.3 (44), 1746.29.5 (49)

- \& a settentrione con la somità di Curnich, che và con Confin di Chiusa. 1749.3.3 (50)

- Curnich. 1749.15.5 (51), 1750.12 .10 (51), 1756.27.2 (124), 1756.5.5 (127-129)

- la somità di Curnich. 1651.6.5 (161)

- ...a piedi dal Colle Curnich tirando per schena del medesimo, o sia, drio Goslò di Gniva, \& Peccolli suso in la somità di esso Curnich, \& indi al confin de Stulvizani, indi passando per altra somità Tolsiurch (!), \& calando per la mezzaria del medesimo a retta linea in Rivo chiamato Crisicinipotoc, confin con Oseachesi, \& al Fiume Resia, \& al precenato Rivo Oragnipotoc. 1654.5.10 (167)

- Curnich. 1668.23.5 (173)

- il bosco drio Goslò ovvero Curnich. 1668.23 .5 (179)

- Curnich. 1718.20.10 (181)

\section{Lástini potök (?) (N)}

- sino per mezzo lo Rivo chiamato Lastiguipatoch, caminando drio due Croce signate in due sassi. 1580.9.6 (14)

40. Lás (N), Lés, Ta-na Lázo $(\mathbf{B})^{34}$

- ...in loco deto Dut Las (!) appresso la Strada Pubblica specialmente dalla parte inferiore appresso il Rivo di Nischipotoch appresso l'Alveo del Fiume Resia. 1499.27.4 (22) [Dut per Dul?]

- ...unum Pratum... in loco vocato Las cum unum Stabulum super posito longitudinis trium Passuum... 1504.14.1 (23)

- in loco ubi dicitur in Las cum Sedimine Stabuli de super posito. 1514.14 .3 (23)

- in loco dicto Las di Tora [per Sora]. 1543.15 .5 (24)

- in loco vocato Las di sopra. 1543.26 .5 (24)

- ...tutto Communale di Las... in passato è stato stato dal Comune di Gniva a risserva delli Prati sotto la Strada che conduce a Resiuta quali sono dalli Particolari di S. Giorgio. 1654.5.10 (167)

34 Non siamo ancora riusciti a decifrare il Dut davanti a Las (1499). Presso uno dei tanti Laz (radure, pezzi di selva disboscata per ottenere prati), entrato nel canto lirico (Da ta na Lazu lipa ma La mia bella di Laz), si noti come da questo toponimo preposizionale è stato ottenuto un aggettivo: Da lipa mâ nalăzawa (Matičetov $1972 n^{\circ} 16$ ). 
- Las. 1654.5.10 (168)

- principiando sotto, e sopra Las di sopra in Scarbina. 1753.25.2 (53)

- neppure nel Monte denominato Bilapech, nè nel Piano denominato Las, il qual Piano si estende dalle radici del Monte sino ai Prati parimenti denominati Las. 1756.27.2 (124)

- Pascoli di Las. $1756.5 .5(127,129,130)$

- Las (Pascolo). 1757.27 .2 (152), 1757.26 .10 (139), 1757.21.12 (143), 1757.31 .12 (146), 1759.18.2 (153)

- Sol a Monte con li Prati di Las. 1777.29.4 (211)

- Prati di Las. 1777.29.4 (212)

- ...principiando dal Fiume Resia, e continuando per il Rivo Nischipotoch sino al luogo di Sagurisizza, dove passate le Colture di Las esistono due grossi Sassi in piano, \& indi passando verso Mezzodì di detti Sassi per il piccolo Rio, e continuando verso Ponente sino a Bilapeg. 1782.9 .7 (311-312)

\section{Lávera (N), Lévara (B)}

- ...Cadin, Lavori per tutte le pendenze di quella banda cadenti in Cernipotoch passando per Goliz per Somità di Pustigost discendendo zoso per Plechie sino al Rivo di Cirnipotoch. 1605.9.1 (75)

- Lavora. 1778.12.3 (219)

\section{Lazones}

- Versus Meridionales Partes est quidam locus vocatus Lazones super Flumen Bible per Mezanesarem. 1382.9.7 (3)

\section{Licinopetoch}

- Licinopetoch. 1382.9.7 (3)

44. Lipovaz - Lïpavac (B) ${ }^{35}$

- Carlo, e suo Figlio Butulo di Lipovaz...1728.20.4 (49)

- dal Lipovaz sino in Porclanaz. 1753.25.2 (53)

- Sopra Lipovaz. 1757.18.4 (153)

- nelli Beni detti volgarmente Sopra Lypovaz, Crisizzis, \& Las. 1757.26.10 (139)

- Sopra Lipovaz. 1757.21.12 (142)

- Gripovaz (!). 1757.31.12(145)

35 Da un canto del vecchio Trankon di Lïpovac: Tau Lipouze ie paravisc / nu ta na Ravanze puclò "A L. c'è il paradiso, / e a Prato l'inferno,..." (Di Lenardo 1974 n. 23). Sarebbe interessante conoscere la ragione di questa dichiarazione del famoso verseggiatore $\mathrm{T}$., ma non ci è stata riferita. 


\section{Lisbine}

- ab Ocidente Rium dictum di Hirmaf, \& Caput Rivi, vocati Lisbine. 1581.6.6 (27)

- ...e il Capo del Rivo nominato Lisbine. 1778.12.3 (220)

\section{Máli kúlk (B), Mćli Kólk (N)}

- ...Selva Pascolo commune, loco detto Malicolch Estosie (!)... 1777.29.4 (211)

- per il Livinal di Meligolch. 1777.29.4 (211)

\section{7. *Máli wárh ${ }^{36}$}

- Et versus partes Occidentales est quidam locus vocatus Malevorch, \& quidam locus vocatus Strop, et quidam alius locus vocatus Misser. 1382.9 .7 (3)

- in montibus nuncupatis Malivorich, \& Strop. 1503.5 .7 (6)

- usque ad Sumitatem Collis, qui dicitur Malivorich, latine vero Pizzol Col, qui autem Collis est prope la Costa delli Larisi, quae vocatur Masatichen Lingua Illirica. 1503.5.7 (7)

\section{Marilla}

- Rivo della Marilla [in un loco drio Goslò, e Curnich]. 1718.20.10 (181)

\section{Masasnátin Klén (?) (N)}

- Collis Malivorich... prope la Costa delli Larisi, quae vocatur Masatichen Lingua Illirica. 1503.5.7 (7)

- Strop, indi traversando in Mis, indi a Masasnati Clin, indi suso alla sommità. $1777.29 .4(212)$

\section{Masësanjë $(B, N)$}

- Bilapeg, con espressa dichiarazione che resti libera la strada per Masessene al Comun di S. Giorgio... per potersi condur, e ricondur con li loro Animali nel sito, o sia Comunali di loro ragione nominati Scarbina. 1782.9 .7 (312)

\section{Mecia}

- Grachicinich..., indi calandosi per il livinale sino in Mecia, o sia Rivo Bianco, indi verso Ponente in Pasigh... 1777.29.4 (212)

\section{Meleznarava ${ }^{37}$}

- versus partes septentrionales est quidam locus vocatus Meleznarava, \& quidam locus Rivus de Licinopetoch juxta Cescamcalle... 1382.9.7 (3)

36 Il primo verso di un canto da Uccea suona: Ziz Kyla ta-na Mali uorh "Dal monte Chila fino al Mali uorh" (Merkù 1976 n 522).

37 A questo nome potrebbe essere avvicinato il toponimo Mlíčna Ráven (O) nella zona di Oseacco, ottenuto dalle fonti orali. 


\section{Mezanesarem}

- Mezanesarem. 1382.9 .7 (3) (cfr. Lazones)

\section{Milli}

- a dicto loco Malivorich veniendo recto tramite per quandam Costam crepidinosam, per prope nemus a parte Superiori usque ad quendum Rivum, qui dicitur Milli, et inde recto tramite ascendendo usque summitatem primi Collis de Strop... a dicto colle de Strop, usque ad ultimun Collem dicti Monti de Strop...usque ad quemdam Lapidem, qui dicitur Miser... 1503.5.7 (7)

\section{Miravolc}

- ...arbitrio presosi da detti Vomini di Gniva d'essersi portati con li loro Animali a pascolare nel sito nominato Miravolc. 1782.7.2 (319)

\section{Mírnik ( $\mathrm{B}, \mathrm{N})$}

- Cominciando da piè del loco detto Mirvich appresso lo fiume Ucea, andando suso per lo Miruhic per diritta Linea, cioè per la Lavinal per sino alla Summità, ovvero cima di esso Miruhic, suso per lo qual Lovinal dissero haver scolpite tre Croce in Sasso, et una in la cima, \& poi da essa cima, over summità per detta Linea andando sino al loco ove nasce lo Rivo Bianco. 1580.9.6 (14)

- ...indi suso per il Mirnich, indi retta Linea suso alla sommità di Grachicinich, ove similmente si trova altra Croce, indi calandosi per il Livinale sino in Mecia, o sia Rivo Bianco, indi verso Ponente in Pasigh, e seguitando per traverso in Zelenipotoch. 1777.29.4 (212)

- Mirnich, o sia Monte Cassaria. 1777.25.2 (214)

- Mirnich. 1779.22.6 (227)

- Il povero Commun di S. Giorgio... ha sempre pacificamente goduto li Pascoli del Monte Casaria nelli siti di Ucea fino al Livinal Mirnich. 1779.15 .9 (229)

- Livinale Mirnich. 1779.15 .9 (229)

- Livinal di Mirnich. 1779.15.9 (230)

- Mirnich. 1780.10.6 (253)

\section{Mis}

- ...da Miziza (!) ...verso Scrimizinadolina... in Strop, indi traversando in Mis, indi a Masasnati Clin, indi suso alla sommità di Sagoldi [per Sagosdi]... 1777.29.4 (212)

\section{Miser/Misia/Misser}

- Misser. 1382.9.7 (3)

- usque ad lapidem dictum miser; qui lapis asseritur esse Confinis principii praesentis Montis Cornice spectantis et pertinentis dicti de Sancto Georgio. 1547.25.6 (10) 
- ...tagli nei Boschi di Sopoludnich, e Braman, siti che cominciano dalla Porta, o sia Restello, e s'estendono fino al sasso chiamato Misia, ovvero Miser... 1722.7 .3 (82)

- ...principiando dalla Porta, sive Rastello, che è a Settentrione sino al Sasso Misser, sive Misiza che è a Levante. 1723.9.11 (95)

59. Mizica $(\mathrm{B}, \mathbf{N})^{38}$

- ...nelli Siti, e Territorio di Braman, e Sapoloduvich [Zapoludnik] promiscui tra loro Communi sino al Confine di Misizza... Moggio, 1721.21.11 (79)

- Sappolodivich, o sia Sapolunich, e Bermant, o sia Bramon, Siti confinanti con Misiza... 1722.22.3 (84)

- Misiza. 1723.9.11 $(96,97)$

- Miziza. 1777.29.4 (211, 212)

- Missisa. $1778.12 .3(220)$

38 Mïzica (panca di legno), in prossimità di un "posto di riposo" (Počiwalca); un canto nella parlata di Ucjà rende bene la situazione: ... se biu taunè na Misizi./ Ia se sednuu sa pucet "...ero arrivato in Misiza./ Mi son seduto per riposarmi" (Di Lenardo $1974 \mathrm{n}^{\circ}$ 18). M. si trova sul vecchio sentiero (oggi strada asfaltata!) che dal Barmán conduce a Njivica. Qualche secolo addietro deve essersi staccata dal monte Kóćaca una imponente frana che raggiunse Mizica, dove ancora giacciono alla rinfusa grandi ammassi di pietre, sbiancate dal sole e dalle intemperie. Un anonimo resiano, dotato di estro poetico, mise l'accaduto in versi, telegrafici ma efficaci: lo staccarsi della frana è paragonato al parto, "partoriente" è la montagna Koćaca, come attori e spettatori di quel "lieto evento" tra le montagne, figurano, personificati, alcuni caratteristici punti geografici dei dintorni. La poesia tradizionale ci ha conservato un originale "certificato di nascita", in cui compaiono in bell'ordine nomi e attività dei coinvolti in quel fantastico scenario: dalla "madre" al comparatico e a quanti, trovatisi lì per puro caso (per es. Ć́bowje), curiosarono, consentendo tutt'al più di essere nominati testimoni oculari, e poi giù giù fino a colui il quale riempì, forse per il rituale brindisi, una coppa di pietra incavata dai vortici del Barmán e trovata anch'essa degna (novello Graal!) di un nome... Per maggiore chiarezza, facciamo seguire qui la lista nominativa di tutti i presenti e dei servigi da loro prestati in occasione di quel famoso parto tra le montagne - povijálo sri gorè:

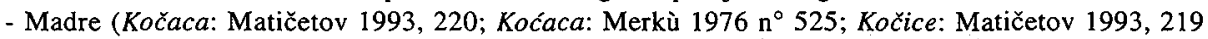
[Silvana Paletti]; Misiza: Di Lenardo $1974 n^{\circ} 51$ ) - Koč. so pövile / Mïz. je pövila.

- "Comadre a levar de terra" (Mizica: Matičetov 1993, 220; Merkù, ibidem) - Miz. je wzdignula.

- Comare di battesimo (Starmica: Matičetov 1993, 220; Merkù, ibidem; Stïrmiza: Di Lenardo, ibidem) - St. šla nünica.

- Compare di battesimo (Zapolúdnik: Matičetov 1993, 220; Zapalüdnik: Merkù, ibidem; Sapulúdnic: Di Lenardo, ibidem) - Zap. je šal / ̌̌il non / nun.

- Balie (Mizice: Matičetov 1993, 219 [S. Paletti]) - Mï. so zibale.

- Comare di cresima (Gniviza: Di Lenardo, ibidem) - Njïv. jo birmala.

- Teste oculare (Č́kowje: Matičetov 1993, 220; Čokouje: Merkù, ibidem) - Čok. kukücalo.

- Coppiere (Studinčé́: Matičetov 1993, 220) - Stud. je wtučil pet.

- Coppa (LonČé́: Matičetov 1993, 220) - <recipiente (passivo)>.

Per finire questa lunga nota ancora un accenno all'importanza che può avere un frammento Ietterario come Kočaca so pövile. Quanti informatori bisognerebbe mobilitare per venire a capo che Kočaca è un plurale! (Nella stessa categoria rientrano probabilmente anche nomi come Krï̌aca, Lǐ̌ćaca e altri). 
- ...fino alli confini di Misiza, spogliando con tal meditazione esso povero Commun di Gniva del Sito Puzzualz intermedio tra li veri Confini del Sito Barmant, e il detto Misiza... 1780.10.6 (253)

- Misizza. 1782.9 .7 (311)

- Missizza. $1780.4 .9(264,265)$

60. $\operatorname{Müzac}(\mathbf{B}, \mathbf{N})^{39}$

- dicti de Resia, \& Gniva pascolare valeant in Monte vocato Musi. 1489.1.7 (21)

- per l'affitto che paga il sudetto Comune all'Tlustr. Consorzio Frangepani per le Montagne di Mussi, e Borgona... 1716.21 .9 (335) [ricevuta per 26 libbre di formaggio]

- aver siegati le Herbe della Monte detta di Musi. 1717.3.8. (54)

- solita Contribuzione, che devon dare annualmente... per le Montagne di Mussi, e Borgogna come compare dall'Affittanza. 1717.26 .8 (335) [ricevuta simile di 26 libbre di formaggio - Giacomo Frangipani]

- Division Monte Mussi. 1717.11.9 (59)

- Vertiva strepitosa Litte... tra l'Onorando Commun di San Giorgio di Resia da una, \& 1'Honorando Commun di Gniva dall'altra... per il Taglio di parte delle Erbe del Monte chiamato Mussi di ragione dell' Illustris. Consorzio Frangipani. 1717.11.9 (59-60)

- Monte chiamato Mussi, che... confina da Levante il Rio Bianco mediante Sasso grandissimo con due Croci segnato, sive Varsaz, Mezzodì il Canal di Mussi, e Pasco goduto dal Commun di Tarcento, \& altre Ville annesse. 1717.11 .9 (60)

- l'inspezione del Monte contenzioso nominato Musi. 1719.9.9. (58)

- altra sommità di Musi, \& drio Musi, di poi a dritta Linea sino in Campo di Cadin. $1777.29 .4(211)$

- Musi. 1777.25.2 (214)

- Monte Musi. 1779.22.6 (224)

- Musi, osia drio Musi. 1779.22.6 (226)

- ...il Communale esistente in Mussi, o sia drio Mussi. 1780.10.6 (251)

- Il Communale Ju mussi [su Mussi?], e drio Mussi. 1780.4 .9 (266)

- Pascolo del Monte Musi. 1781.6.3 (278)

39 Stando a una recente informazione $(O)$, i vecchi resiani avrebbero usato per questa montagna il nome generico di Plahüte, ossia lenzuola (Ta-s te Plahüte). Questo termine si adatta bene al ripido versante settentrionale dei Musi, dove - chissà quando - valanghe e lavine asportarono terra e vegetazione lasciando scoperti grandi lastroni di roccia viva, ben visibili da lontano. Nel 1962 , invece, il nome Plahüta in una leggenda sulla Hüda Ura, cioè la Tempesta personificata (B), sembrava usato non come termine generico, bensì locale, riferito a un determinato lastrone, sulla destra del Barman, in prossimità del vecchio sentiero che portava a Karnïca /Carnizza. 
- il Monte Musi nominato Barghin, sive Stermas, che riconosce detto Comun di S. Giorgio con titolo separato dalla Casa Frangipani, e che è separato dall'altra parte di Musi dipendente da Titolo d'ambidue li Comuni, e che viene posseduto promiscuamente, abbia a rimaner di detto Comun di S. Giorgio, \& esso solo debba possederlo senza ingerenza immaginabile del Comun di Gniva. 1782.9.7 (312)

\section{Niski patök (B) ${ }^{40}$}

- appresso il Rivo di Nischipotoch appresso l'Alveo del Fiume Resia, appresso un certo Condutorio, o sia Manadore di Legni. 1499.27.4 (22)

- Rivo Nischipotoch. 1777.29.4 (212)

- Rivo di Nischipotoch. 1778.12.3 (219)

- Niscipotoch. 1778.12.3 (219)

- oltra il Rivo di Nischipotoch. 1780.10.6 (254)

- oltre il Rivo Mischipotoch. 1780.4 .9 (265)

- Rivo Nischipotoch sino al luogo di Sagurisizza. 1782.9 .7 (311)

62. Njivica: Ta-w Njivici (B), Tu-w Njïici $(N)^{41}$

- ...super loco Montis Cranize Gnivice. 1547.25.6 (9)

- ad locum certae Vallis parvae vocatae Gnivicae. 1547.25.6 (10)

- le Montagne di Gnivizza, e Carnizza di ragion di Gniva che cominciano dall'accennato confine Misiza... 1723.9.11 (96-97)

- Pendenze di Carniza, e Gniviza. 1723.9.11 (97)

40 L'informatore (B) conosce un Niski patök nella zona di Oseacco.

41 Il nome qui non è un semplice dim. di njiwa (campo), come nel caso del villaggio Njivica (Vedronza) nell'alta val del Torre, a sud di Ter (Pradielis), com. di Bardo (Lusevera). La nostra Njïvica (Nella zona di Karnica/Carnizza) è un diretto rampollo, una "succursale" del villaggio resiano Njiwa, una sua proiezione oltre lo spartiacque tra Tagliamento e Isonzo. Ci troviamo di fronte a una tipica stazione di transumanza estiva, dove il legame di figliolanza è corroborato dai nomi: Njïwa (madre) e Njivica (figlia). In mancanza di precisi dati sull'origine e lo sviluppo storico di Njivica sarà meglio ascoltare la leggenda che, tra l'altro, spiega il perché di certe anomalie nella divisione dei pascoli montani (cfr. Matičetov 1968-71). Alla Njüvica di una volta davano il tono di villaggio anche gli osti che potevano servirsi, in montagna, della "licenza" loro concessa per l'esercizio a valle. Il terremoto del 1976 diede però il colpo di grazia al lento processo di abbandono in atto già prima. La poesia popolare resiana ci offre quasi un inno al curioso "locum certae Vallis parvae vocatae Gnivicae" (1547) che, o per ragioni prosodiche, o semplicemente perché "variatio delectat", diventa ora lipa moja Njyvica ora mâ te lipa Njyvica (O cara mia Gn. Matičetov $\left.1972 \mathrm{n}^{\circ} 11\right)$. L'itinerario fittivo di un giovane innamorato, in cerca della sua bella di Učjà, parla di una sosta anche a Njüvica: Co se dusciou uon Gnivizo, / ia se ućiutel zittiro (Quando sono arrivato a Gnivizza, ho sentito il suono della citira - Di Lenardo $1974 \mathrm{n}^{\circ} 18$ ) 


\section{Obláze: Ta-dö z Obláze (K), Wobláze (B) ${ }^{42}$}

- primo dalla Forame di Obblas fino in Sagatte tanto d'Erbe vive, quanto d'Erba morta. 1667.16.1 (38)

- il Pascolo di Oblaes. 1680.28.3 (44)

- Oblas. $1749.15 .5(51), 1753.25 .2(53)$

\section{Pasigh}

- Grachicinich..., indi calandosi per il Livinale sino in Mecia, o sia Rivo Bianco, indi verso Ponente in Pasigh. 1777.29.4 (212)

\section{Përaćace $(\mathbf{B})^{43}$}

- Monte Periachiaz. 1654.5.10 (166)

- Perachiaz. 1654.5.10 (167)

- Parachiaz. 1749.3.3 (50)

- Perachioz. 1749.3.3 (51)

42 Nel tempo tra la prima (1667) e l'ultima (1753) testimonianza scritta tale nome era attribuito a tutta la pendice, dal fondovalle sulla destra del Bila e su fino al margine Sud dell'altopiano - "fino in Sagatte" (Ta-wnë w Zagate) ed è probabile che ancora oggi sia così. Appena quando negli anni trenta del sec. scorso fu trasferita sulla destra del Bila la carrabile Resia-Resiutta e da Podklanac in là la strada venne a trovarsi alle falde della zona Oblaz, questo nome cominciò a entrare nella coscienza di un maggior numero di resiani e non solo di quelli dei vecchi comuni di Bila e Njüwa. Fu così che il tratto di strada che lambisce Oblaz e va verso il confine comunale resiano, segnato da una majana o cappella detta "Salveř̌ina", ebbe pian piano il nome "Ta-z Oblázi", passaggio obbligato di tutti $\mathrm{i}$ resiani che se non per sopravvivere almeno per guadagnarsi da vivere meglio dovevano "andar via" (jtet pröč), cioè emigrare. Döz Obláze pòti mê, / tu ka mi öci kážejo... (B - Le mie strade giù attraverso O., là dove mi mostrano gli occhi - Matičetov 1978, p. 61). Così si può capire perché il poeta Renato Quaglia a una della sue poesie diede il titolo metaforico di "Ta Oblazawa", che nella traduzione slovena letteraria di Marko Kravos suona "Pot v svet" (R. Quaglia, Baside, Trst, 1985, pp. 66-69).

43 Delle due forme trasmesse per iscritto, quella masc. sg. compare per prima (Perachiaz, 1654), tuttavia non possiamo dichiararla più attendibile di un posteriore Peragiazzà (1777), specialmente perché quest' ultima vive nella tradizione orale, mentre di Perachiaz non si trova traccia. Può darsi che, se non si tratta di una (senz'altro falsa!) analogia con Lipovaz e Porklanaz (che compaiono fra l'altro anche assieme in uno stesso documento del $25 \mathrm{feb}$. 1753) sia da considerarsi una risposta "Zis Përaćac" - alla domanda "da dove vieni?". Il tentativo di spiegare "Peračaci"(!) come "porticina" e di accostarlo nientemeno che a una parola turca (v. Matičetov 1993, p. 68, $n^{\circ} 45$ ) è un esempio tipico di etimologia affrettata e subordinata a teorie preconcette. Come eventuale parallelo a Përaćaca (con accento sulla prima sillaba) si potrebbe accostare il nome di un luogo (non abitato) della Carniola superiore (Gorenjsko): Pirecica (viadotto all'uscita $O$ dal traforo della strada tra Ljubno e il santuario di Brezje). Da gentile informazione orale del dr. Dušan Čop, esperto di toponomastica della zona, si viene a sapere che il vero nome del torrente in fondo alla forra sopra la quale si erge il viadotto non è Pirečica ma Peráčica (fem.sg.) e che nomi analoghi esistono anche nella conca di Bohinj e altrove in Slovenia. Il Nom.pl. p'øraţats $\emptyset$ si trova anche in un piccolo prospetto tipologico dei nomi di luogo resiani (O. Kronsteiner 1975, $119 \S 2.2 .1$ ), assieme a kr'y $\oint \varnothing t s \emptyset$, -pl' $\emptyset t ̧ a t s \emptyset$, put $\int u^{\prime}$ 'alits $\emptyset$ e sim. (con qualche incongruenza grafica; a titolo di esempio abbiamo scelto solo nomi che conosciamo dal nostro elenco). 
- Perachiaz con li Pascoli attorno la Tavella dal Lipovaz fino in Porclanaz. 1753.25.2 (53)

- Peragiazzà. 1777.29.4(210)

- Peragiaz. 1777.15.2 (214)

- Perachiaz. 1779.22.6(225)

\section{Petro Fartivo}

- Confinante... a Septentrione cum Petro Fartivo, ac claputii prope primum Albam. $1614.18 .8(36)$

\section{Plaćë $(B, N)$}

- Item un'altra Pezza in loco, che si dice Plechie. 1609.16.8 (32)

- Plegie. 1680.28.3 (44)

- Plachie. 1726.9.4 (47)

- Plegie. 1749.15.5 (51)

- Inplechie. 1753.25.2 (53)

- Plechie. 1778.12.3 (217)

\section{Planinïca (B), Plininïca (N)}

- per la Scrassa di Planiniza, \& Stansissa... 1605.9.1 (75) (rinnovazion Bando 1597)

- Monte di Planiniza. 1622.26.7 (76)

- Monte Planiniza. 1622.26.7 (77)

- attraversando sotto Planiniza s'estendono fino al Luoco detto Billipeg, e Cirnipotoch. 1723.9.11 (95)

- Planiniza. 1723.9.11 (97)

- ...pascoleggio degli Animali... fatti condurre nel Monte Planiniza. 1775.27.7 (356)

- Animali del Monte Planiniza. 1775.27.7 (357)

- pretesi Confini delli Monti Cernipotoch, e Planiniza. 1776.26.6 (360)

- la verità fù, \& è, che Scrassa di Planiniza dalle Carte di Confini Chiamata, è sempre stata, \& è identicamente la situazione, e pendenza Credosa, che hà la sua Pianta, e Radice nel Monte di Planiniza... 1776.26.6 (361)

- Scrassa di Planizza. 1777.25.2 (214)

- Scrasa di Planneniza. 1777.29.4 (211)

- la Planiniza di ragione degli Abitanti del Commun di Resiuta. 1777.29.4 (212)

- la Monte di Planiniza. 1777.27.5 (363)

- Planinizza. 1777.27.5 (363)

- La verità fù, \& è, che la Parola Sgrasa in Lingua Illirica, o sia Reseana significa in Italiano Sfesa, o sia Schiapadura. 1777.7.6 (362) 
- andando suso in Cuzzer, discendendo poi in Scrassa di Planinizza, o sia Forcastina, andando sino alla sommità del Monte Musi. 1778.12.3 (219)

- Sclaza di Planinizza. 1779.22.6 (224)

- Scrassa di Planizza. 1779.22.6 (226)

- Scraza di Planinizza. 1780.10.6 (252)

- Scraza di Planinizza. 1780.4 .9 (266)

- Scrazza di Planinizza. 1781.6.3 (279)

- Scraza di Planizza. 1782.9 .7 (312)

\section{Polúdnik/Pulúdnik (B), Pulúdnik (N)}

- ...in facie Solis in loco vocato Polunich. 1636.11.7 (337)

- ...sopra Sappolodnich additando verso la Cima d'essa Pendenza il sito chiamato Palunich a distinzion di Sappolodnich che stendesi al di sotto e a differenza pure di Billipeg, che è l'opposta Pendenza di detto Colle, che scende dall'altra parte sopra il Fiume Resia. 1723.9.11 (96)

- Poloduich. 1723.9.11 (97)

- Negando espressamente, che il Termine di Poludnich sii diverso da Sappuludnich nè che importi diversità di sito come vorrebbe capziosamente disseminare a studio di equivoco. 1723.9.11 (99)

- Sapolodovich, o sii Palvich. 1725.13.1 (112)

- negando che i Siti di Sapolodnich, e Palunich siano lo stesso.1736.1.12 (342)

- [il Comune di Gniva interpella il Comune di S. Giorgio] a confessar se entro i Confini descritti nell'Investitura 1636 11. Luglio del Sito denominato Palonich sii compreso anco il Sito detto Sappolonich... 1736.15.12 (344)

- ...rispetto alla mal professata indentità di Puludnich, e Sapaludnich. 1737.12.1. (345) [rimanda al suo punto di vista espresso il 9 nov. 1723]

- dichiaro... che sia, e s'intenda il Sito controverso entro i Confini del Investitura 1636. 11. Luglio di Paludnich, ovvero Sapoludenich, e particolarmente sino al Rivo Sighipotoch di ragione del Comun di Gniva... 1739.9.5 (347) [Sentenza a favore di Gniva]

- Puludinich. 1749.15 .5 (51)

- Puludivich ovvero Storimlin. 1753.25.2 (53)

- Paludrich. 1777.29.4 (212)

- La sentenza del dì 9 Maggio 1739 ... ha malamente, ed ingiustamente giudicato, che il Sito denominato Sappulodnich sino al Rio Sachipotoch sia di ragione del detto Comun di Gniva. 1781.9.4 (349)

70. Potklánac, Ta-pot Kláncon (B)

- Porclanaz. 1753.25.2(53) 


\section{1. *Potök $\mathbf{k}^{44}$}

- dal Ponte Resia per il Rivo detto Potoch. 1777.29.4 (211)

\section{Pradolína (N), Ta-na Pordúlinëj (K)}

- Montem qui vocatur Prodolina. 1382.9 .7 (2)

- Stare Starnisca, e Pradolina Monti contigui, e Pascolo apparente (sic!) alli Monti predetti. 1778.12.3 (219)

\section{Pučuwálca, Tu-wnë na Pučiwálcəh $(K)(?)^{45}$}

- ...del Sito Puzzualz intermedio trà li veri Confini del Sito Barmant, e il detto Misiza... $1780.10 .6(253)$

- Puzzualz. 1781.13.2 (264)

- Puzzualz, che esiste tra li... Confini del sito Barmant e quelli di Missizza.1780.4.9 (265) [così sul foglietto a stampa incollato sopra la forma scorretta Duzzualz]

- Puzzualz. 1781.13.2 (300)

- li Comunali detto Barmant, e Pozualz sino in Misizza. 1782.9.7 (311)

\section{Pulice (B)}

- quel sito preciso...dietro Goslò... e che viene per maggior specificazione denonziato Pollice. 1757.27.2 (151)

- ...nè possa poner mano, e praticar tagli in quel sito preciso denominato dietro Goslò, ...e che viene denominato anco per maggior specificazione, Polizze parte integrante del sudetto sito. $1757.31 .12(145)$

\section{Püsti Göst (B) ${ }^{46}$}

- passando per Goliz per Somità di Pustigost discendendo zoso per Plechie sino al Rivo de Cirnipotoch. 1605.9.1 (75) (Rinnovazion Bando 1597)

- fino drio Pustigast (!), sino Bresaviza... 1778.12.3 (219)

44 Potök/Patök in resiano sta per 'torrente'. In Resia. Numero unico, p. 74, il Rivo Potoch (1672) corrisponderebbe al torrente che scorre tra Oseacco e Gniva che dagli uni viene chiamato Osöjski potök 'torrente di Oseacco', dagli altri Njüvaški putök 'torrente di Gniva'.

45 Questo nome, che significa "posto di riposo", si riferisce anche ad altre località della valle; la più famosa sarebbe forse Pučiwalca (S) che si trova alle pendici del Sart dove per parecchio tempo è vissuta la fam. dei క̌enovi-Skwerč (S). BdC nei Materiali I $\S 509$, contiene probabilmente il toponimo di cui si fa menzione nel libro delle liti, poiché, in nota a Pučiwálca sta scritto "dove riposano andando per Carnizza".

46 Gli informatori interpellati conoscono solo la località-altopiano Püsti Göst (B)/Püsti Öst (S) a nord di Stolvizza. Di questa località una volta si cantava: Du bei pussical Pustigost, / vis Sart nu usò Indrinizo, / nu dardu una Përauo? "Chi ha falciato P., / tutto il Sart, tutta Indriniza / e fin sul picco Përauo?" (Di Lenardo $1974 \mathrm{n}^{\circ} 26$ ). 


\section{Rávančin Kúlk (B)}

- Item altra Pezza in loco chiamato Ravanzinculch appresso la strada Publica, ed il Pascolo Commune. 1609.16.8 (32) [Investitura]

- altro pezzo chiamato Ravanzinculch. 1726.9 .4 (48)

- Revanziaculch.1777.12.3 (218) [trascrizione errata delle due forme precedenti]

\section{Rebenich}

- ...item alia Bona Comunalia in Monte vocato Rebenich ab Ortu juxta Prata dictorum de Gnive, a Meridie Rivum Sichipoch, ab Ocasu locum de Belipeg. 1636.11 .7 (337)

\section{Reisdainscaora}

- Monte Reisdainscaora, e Paludrich, principiando a Sol a Monte col Rivo Nischipotoch. 1717.29.4 (212)

79. Rép: Ta-na Répo, Ta-wnë w Répo (B) ${ }^{47}$

- Item altra Pezza in Reep appresso li Eredi qu: Zuanne Palet, ed il Pascolo Comune, e Strada Pubblica. 1609.16.8 (32)

- altro pezzo in Rep. 1726.9.4 (48)

- Reep. 1778.12.3 (218) [Investitura; data corretta a penna: 177727 febbraio]

80. Resenebordó ${ }^{48}$

- in Scarbina per Cuzer per Resenebordò. 1749.15.5 (51)

\section{Resiute - Resiutta - Ta-na Bíli (B)}

- Resiuta. 1654.5.10 (167) (Accordo querelato)

- Risciuta. 1660.1 .5 (172)

\section{Rio Bianco - Bíli Potök (U)}

- Rivo Blanco. 1382.9 .7 (3)

- ove nasce lo Rivo Bianco. 1580.9.6 (14)

- Rivo vocato Rivo Alvo. 1581.29.5 (25-26)

- a Montibus Rium album. 1581.29.5 (27)

- confina da Levante il Rio Bianco mediante Sasso grandissimo con due Croci segnato sive Varsaz. $1717.11 .9(60)$

47 Nella valle ci sono anche altre località con lo stesso nome Rep, che designa una striscia di terreno oblunga.

48 Questa forma potrebbe essere in rapporto con orësje 'erica' e brdo. 


\section{Rischipotoch ${ }^{49}$}

- ...a Ponente il Rivo Rischipotoch suso in la sumità detta Sia ovvero Rut...1654.5.10 (166)

\section{Rop: Ta-h Róbu (N)}

- caminando [dalla Monte Casaria/Karnïca] suso alla sommità di Rop seguitando altra sommità di Musi, \& drio Musi,... di poi a dritta Linea sino in Campo di Cadin. $1777.29 .4(211)$

85. Rüšće (B) ${ }^{50}$

- Ruschis. 1753.25.2(53)

\section{Rüt (B)}

- ...in la Sumità detta Sia ovvero Rut. 1654.5 .10 (166)

\section{Scrimizinadulina/Serimizinadulina ${ }^{51}$}

- Livinal di Meligolch, seguitando per la Sommità sino in Serimizinadulina, indi calandosi a Miziza, che confina colla Monte Casaria di esso Commun di Gniva...1777.29.4 (211)

- principiando da Miziza in suso Scrimizinadolina, indi suso Strop... 1777.29.4 (212)

\section{Slonziplas/Slorigi Blas}

- ab oriente Lavinam dictum de Slorigi Blas. 1581.6.6. (27)

- confina a Oriente Lavina detta Slonziplas, a Mezzodì Sommità del Monte, che è sopra il Monte nominato Maggior, a Occidente il Rio detto Stormaz, e il Capo del Rivo nominato Lisbine, ed a Monte il Rio Bianco. 1777.12.3 (220)

\section{Stér mlán (B)}

- Item un altra Pezza in loco detto Starimilian, presso il Pascolo del Commune, appresso i Cretti. 1609.16.8 (32)

- altro pezzo chiamato Starimlin appresso il Pascolo, appresso i Creti... 1726.9.4 (47)

- Puludinich, o sia Storimlin. 1749.15.5 (51)

- Puludivich, ovvero Storimlin. 1753.25.2 (53)

- Starimilian. 1778.12.3 (218) [Investitura; correzione a penna: 177727 febbraio]

49 Probabilmente si tratta del Bíski patäk che rappresenta il primo tratto, a monte, del torrente che scorre attraverso Bila, dove viene chiamato invece Wuznik (B).

50 Il nome della sede di transumanza estiva Ruschis è scritta Rusćie (Di Lenardo $1974 \mathrm{n}^{\circ} 10$ ), Pa ta na Ruscie bei ni scil "Anche a R. non andrei" (Id. n 11), Rǚće (Merkù 1976 n. 541) o Rüšća (Id. n 544); l'agg. possessivo nel Nsg f. è Rǚ̌cina (Merkù $1976 n^{\circ} 541$ ).

51 In Resia. Numero unico, 1967, a p. 74 compare Smirizina Dolina che ci permette così di interpretare il toponimo con più facilità, riconducendolo a smerëka/smrëka 'abete' e a dolïna 'valle'. 


\section{Stármac (O) (pascolo)}

- Pascum nominatum... Barghin, sive Starmaz. 1581.29.5 (25)

- un Pasco della Monte di Musi chiamato Barghin, ovvero Starmaz. 1717.2.9 (57)

- Item il Pascolo del Monte Musi, ...nominato Barghin, sive Stormaz.1778.12.3 (220)

- Barghin, sive Stermaz. 1781.6.3 (279)

- il Monte Musi nominato Barghin, sive Stermas. 1782.9 .7 (312)

90.a *Stármac (rio)

- ab Ocidente Rium dictum di Hirmaf, \& Caput Rivi, vocati Lisbine. 1581.6.6 (27) [nel documento originale forse compariva al posto di Hirmaf la forma Stirmas, con s lunga]

- a Occidente il Rio detto Stormaz, e il Capo del Rivo nominato Lisbine. 1778.12.3 (220)

91. Starnî̌śé $(B, N)$

- Montem qui vocatur Stare Stranisca. 1382.9.7 (2)

- habentes partem vocatam del Miruhic habeant, \& habere debeant liberum accessum, $\&$ regressum cum eorum Animalibus, \& Gregibus ad dictum Montem per stratam, qua tenditur ad locum appellatum Starnischia. 1580.9.6 (15)

- Homines villae Gnivae... elegerunt sibi partem, et portionem Montis Inferioris, qui vocatur de Starnischia. 1580.21.6 (16)

- Se in Starnischia e Cirnipotoch Montagne di Ragione di San Giorgio siino Boschi di Fagaro. 1723.9.11 (95)

- la Montagna di Starnischia stendesi fin al Fiume di Uva [= Učja] verso Plez. 1723.9.11 (97)

- Starnischia. 1723.9.11 (98)

- Stare Stranisca, e Pradolina, Monti contigui. 1778.12.3 (219)

92. Stolvise - Sólbica

- Piero Negro nativo di Stolvise... 1722.17.10 (89)

93. Strop $^{52}$

- Strop. 1382.9.7 (3)

- indi suso in Strop. 1777.29.4 (212)

- Strop. 1503.5 .7 (6)

- usque summitatem primi Collis de Strop... in Cacumine dicti Collis, ... in loco eminentiori ad evidentiam ipsorum Confinium in aliquo Lapide Congruo celari

52 In BdC 1895, § 508 compare un toponimo Tána Ströpó, che dovrebbe corrispondere alla forma antica riportata in elenco. 
debeant Signum Crucis... usque ad ultimum Collem dicti Monti (!) de Strop in quo Colle alia Crux celanda sit. 1503.5.7 (7)

94. Studeniz 53

- ...a fonte de Studeniz. 1613.24.6 (34) [si riferisce alla 'Concessionem...de Anno 1598.1.5']

95. Sühi Potök (N)

- Rium Sichipotoch; Rivum Sichipoch 1636.11.7 (337)

- particolarmente sino al Rivo Sighipotoch. 1739.9 .5 (347)

- [Bassura di Forchia], indi discendendo, e caminando per il Rivo Suchipotoch sino in altro Rivo Barnian. 1777.29.4 (211)

- Suchipotoch. 1779.29.4 (212)

- Suchipotoch. 1777.27.5 (365)

- per il Rivo Suchipotoch fino in altro Rivo Barman. 1779.22.6 (226)

96. Šija (B)

- in la Sumità detta Sia ovvero Rut. 1654.5 .10 (166)

97. Škarbïna (B), wón zis Škarbïno (L) ${ }^{54}$

- nel Monte Discarbina; in cima la Monte Scarbina. 1605.9.2. (75) (Rinnovazion Bando 1597)

- Item in altro Pezzo sopra il Monte di Sgarbine, in loco chiamato Tanavosepeg appresso il Pascolo Comune d'ogni intorno. 1609.16.8 (32)

- de nemore Scarbina. 1613.24.6 (34)

- super Montem appellato Sgarbina... 1614.16.8 (36)

53 Tale toponimo è piuttosto frequente nella valle. Il significato originario, che è quello 'sorgente', non è più conosciuto perchè è stato sostituito da mlaka (cfr. anche Matičetov 1992, 91). Tale supposizione è avvalorata dal fatto che, secondo gli informatori, in tali località sgorgano delle acque, per es. Ta-par Študunčiću/Štuluncḯcu (B), Tu-w Studince (O).

54 Un canto resiano registrato a Bila-S. Giorgio nel 1962 inizia così: Da jöra mâ Škarbinina,/ ka na ma štiri rožice... (Matičetov $1972 \mathrm{n}^{\circ}$ 27). "Quel monte di Š. / che ha quattro fiorellini...". Però trasposto nel linguaggio comune, di ogni giorno, quest'inizio suonerebbe: "Il villaggio di Bila ha quattro ragazze da marito." Una vecchia legge poetica resiana infatti non permette di dire apertamente il nome del villaggio del quale si vuole cantare, ma suggerisce di ricorrere a certe metafore ben conosciute da tutti. Siccome di fronte a Bila-S. Giorgio sta il massiccio monte Škarbina, per un resiano basta sentir nominare $\breve{S}$. che già sa di cosa parla questo canto: di Bila. Lo stesso potrebbe dirsi, per es., in merito ai canti della Dorina Di Lenardo 1974 n. 1, oppure di P. Merkù $1976 n^{\circ}$ 542: Attenzione! Giacché la battuta iniziale, il primo verso accenna a Škarbüna, sentiremo senz'altro parlare di Bila e quindi, con la mente, trasferiamoci subito a Bila! Più o meno lo stesso si potrebbe dire anche per altri paesi e frazioni della valle: Kucëra = Lišćaca, Sart = Solbica, Ćanen = Korïto, Banëra = zona di Jama... Non dobbiamo naturalmente esagerare, generalizzare; l'argomento comunque richiede di essere approfondito. 
- Sgarbina. 1680.28.3 (44)

- altro pezzo sopra il Monte Sgarbina; altro pezzo pur sopra detto Monte in loco chiamato la Tasasgarbina. 1726.9.4 (47)

- in Scarbina per Cuzer per Resenebordò. 1749.15.5 (51)

- Las di sopra in Scarbina. 1755.25.2 (53)

- Monte di Sgarbine. 1778.12.3 (217)

- Scarbina. 1782.9.7 (312)

98. Škráža (B)

- Scrasa. 1777.27.5 (365)

- Crassa. 1777.27.5 (365) [Zuanne qu: Domenico Beltrame di Resiutta testimonia di aver pascolato per 18-20 anni in quel di Planinica, dove si trova questa Skraža/Costone (363-365)]

\section{9. Štáncije, Štáncje (N)}

- ...\& Stanssissa caminando via per Cadin, Lavori... 1605.9.1 (75) (Rinnovazion Bando 1597)

- Stanzis. 1723.9.11

- e di San Giorgio Cernipotoch compreso il Sito chiamato Stanzis. 1723.9.11. (97)

- Cadin, indi suso per la Sommità di Stante, discendendo in Scrasa di Planneniza. 1777.29.4 (211)

\section{0. Štožjë (B), Štužjë (N)}

- Malicolch Estosie. 1777.29.4 (211)

\section{Támor, Ta-par Támoru (B), dö par Témoru (L) $)^{55}$}

- usque ad Summitatem dicti Montis Cragnice, in quo loco possit (!) sunt duo Tamaci, unus scilicet, \& primus pro usu Animalium dictorum de Gniva, \& alius, scilicet, secundus, pro usu Animalium dictorum de S. Georgio parum distantes a certo riuvulo, sive Agare, sive Aqua tunc dividenti dictam partem Montis Cranice spectante dictis de S. Giorgio ab alia parte superiore dicti Montis pertinentis dictis de Gniva... 1547.25.6 (11)

55 Relativamente al Tamor, da una poesia registrata a Ucjà l'ultimo giorno di Carnevale 1969, riportiamo il seguente passo: Mi ceuna jte nu sforina / nu rauno dö par Tämoro / ziz no karëto garmouso (Merkù $1976 \mathrm{n}^{\circ}$ 524). L'ignoto poeta che, come tutti i montanari di vecchio stampo (dall'alta valle dell'Isonzo fino alla Carnia) usciva sempre con una bella gerla (rez. korba, frl. carnico còsse) sulla schiena, intrecciata con rami di nocciolo (garm), così caratteristica che appare perfino sulla copertina della raccolta Merkù, si è divertito (a Carnevale ogni scherzo vale) a chiamare questo suo "mezzo di trasporto" ironicamente karëta... Pertanto proponiamo una nuova lettura del passo in questione: Io partirò e me ne andrò / dritto giù al Tamor / con un carretto di nocciolo (ossia con la gerla sulla schiena). 
- a lapide praedicto miser (!), usque ad Tamacos. 1547.2.7 (12)

- ...tutto il Piano, ch'è nella Radice del Monte Planiniza con tutto il Pecol sino al primo piano del Monte istesso dove si dice il primo Tamoro... 1622.2.8 (77)

- restando in detta parte ad uso di detti di Gniva il Tamoro chiamato del Pozzo, ovvero Bramischiblas. 1622.2.8 (77-78)

\section{Tanavosepeg}

- Sopra il Monte di Sgarbina, in loco chiamato Tanavosepeg appresso il Pascolo Comune d'ogni intorno. 1609.16.8 (32)

- in luoco chiamato Tanavosepeg. 1726.9 .4 (47)

- ...sopra il Monte di Sgarbine, in luoco chiamato Tanavosepeg. 1778.12.3 (217)

\section{Ta-za Škarbüno (N)}

- Item un altra Pezza sopra detto Monte in loco cognominato Tasasgarbina appresso da tutti i lati in Pascolo Commune. 1609.26.8 (32)

- Tasasgarbina. 1778.12.3 (218) [copia della trascrizione del 1609]

\section{Tölska Stína / Törska Stína (N) - Torsca Stigna}

- ...al luoco chiamato la Forca nel sito poi capitolato, hò sentito qualche volta da Pastori stessi chiamar Torsca Stigna, ma precisamente non mi arricordo, interrogati poi questi stessi perchè chiamassero detto sito in due forme, risposero in sostanza significar l'istesso tanto uno, che l'altro termine, ma esso indifferente il termine per l'espressione della Lingua. 1777.27.5 (364)

\section{5. *Tolsti Vrh - Túlsti Wár (B)}

- Tolsiurch. 1654.5.10 (167)

- Tolstiurh o sia Goslò. 1757.27.2 (151)

- del Monte sopra Lipovaz, e Tolstiurh, o siano Goslò, \& sino alli Confini di Oseacco... 1757.21.12 (142)

- Tolstiurch o sia Goslò. 1757.31.12 (145)

- Tolsti Uvar. 1777.29.4 (210)

\section{Trabnibardò}

- Trabnibardò. 1750.12 .10 (52)

\section{Trabnilidùi ${ }^{56}$}

- Trabnilidù drio Goslò. 1749.15 .5 (51)

56 La seconda parte del composto, il -dù finale (da Dol/Dul "vallone"), può esistere anche autonomamente, come lo dimostra il $D u l$ al margine N di Osoane, scomparso nel 1976 quando le ruspe vi hanno spazzato dentro i resti del più grande villaggio resiano. Forse di qua a mille anni gli archeologi suderanno per riportare alla luce $\mathrm{i}$ numerosi ed interessanti elementi architettonici in 


\section{Učjà - Uccea}

- ...Stare Stranisca..., Prodolina..., Chilla, omnes Montes contiguos, \& ad invicem se tenentes, \& Pascuum pertinens ad Montes praedictos et quemlibet ipsorum, cum omnibus juribus, \& actionibus realibus, \& personalibus ad Montes praedictos, \& in eorum quemlibet spectantibus \& pertinentibus sitos, \& positos in loco dicto Canale de Vicca, \& in Rivo Blanco... 1382.9 .7 (2-3)

- la Montagna di Starnischia di ragion di San Giorgio che stendesi sin al Fiume Uva verso Plez. 1723.9.11 (97)

- Stare Stranisca, e Pradolina, Monti contigui, e Pascolo apparente (!) alli Monti predetti con tutte le azioni alli Monti predetti appartenenti situati, e posti in luogo detto Canal di Ucea in Rio Bianco. 1777.27.2 (219)

- nelli siti di Ucea... M. Casaria, Livinale Mirnich. 1779.15 .9 (229)

\section{Varsaz}

- [Divisione Monte Mussi:] conferiti... sopra il prenominato Monte chiamato Mussi, che unito, confina da Levante il Rio Bianco mediante Sasso grandissimo con due Croci segnato, sive Varsaz, Mezzodì il Canal di Mussi, e Pasco goduto dal Commun di Tarcento, \& altre Ville annesse, Sol a Monte la Spetabil Communità di Gemona, o Venzone, \& alle Monti la Fabrica della Rever. Abbazia di Moggio per mità della Cima d'esso Monte. 1717.11.9 (60-61)

- sive Varsaz. $1717.11 .9(61)$

\section{Wránji patök / Ránji patök $(B)^{57}$}

- Rivo di Uragnipotoch. 1651.6.5 (161)

- Rivo Uragnipotoch. 1654.5.10(166)

- Uragnipotoch. 1654.5.10 (167)

- Rivo Oragnipotoc. 1654.5.10 (167)

- Rivo Uragnipatoc sino alla sommità del Monte sopra Lipovaz; Rivo Uragnipotoc. 1757.21.12 (142)

- Rivo Uragnipotoc. 1757.31.12 (145)

- Rivo Uragnipotoch. 1757.27.2. (151)

- Loco vocato Uragni Potoch...Uragnipotoch. 1777.29.4 (210)

pietra della vecchia Oso(j)ane. All'ultima padrona del Dul, Pasqua Siega "taw Dúle" o, tout court, Paska Dúlica, morta prima del "potrès", fu risparmiato il cordoglio di vederne la rovina.

57 Accanto all'interpretazione a) "Torrente dei corvi" ne girano nella valle altre due: b) Rejni patok $(\mathrm{R})$, cioè torrente morto "dove abitano le volpi. Molti ma molti anni fa lì non scorreva acqua e nessun cacciatore e nessun cane osava andarci" (M. Matičetov, Ce fastu?, 50-51, 1974-75, p. 112); c) Réjnik potök 'torrente (del) defunto', perché secondo una leggenda (O), in quel luogo sarebbe stato ucciso un prete. 
- Uragnipotoch. 1779.22.6(225)

- Uragnipotoch. 1780.11.3 (238, 239)

- ...due Rivi Uragnipotoch, e Brusignipotoch... $1780.11 .3(241,243)$

- Uragnipotoch, e Brusinipotoch. 1780.18.4 (246, 247)

111. Zagáta: Ta-wnë w Zagáti (B) ${ }^{58}$

- in loco dicto in Sagatta. 1558.31 .5 (30)

- in loco chiamato Sagata. 1609.16.8 (32)

- la forame di Obblas fino in Sagatte. 1667.16.1 (38)

- Sagata con suo Pascolo drio Goslò. 1680.28.3 (44)

- Sopra Sagata in loco detto drio Goslò. 1749.3 .3 (50)

- Sagata. 1749.15.5 (51)

- Rivo Sotto Sagata. 1750.12 .10 (52)

- Segata. 1753.25.2(53)

- Sagatta. 1778.27.3 (217)

112. * Zagorï̌ica ${ }^{59}$

- Andando in suso per Rivo di Nischipotoch sino al loco di Saguriziza, indi al sasso di Bilapeg... 1778.12.3 (219)

- Montagne Cernipotoch sino al luogo di Saguriziza... 1780.10.6 (254)

- Montagne Cernipotoch sino al luogo di Sagurisiza. 1780.4.9 (265)

- Sagurisizza. 1782.9.7 (311)

113. *Zagoslò: Ta-za Goslón (B)

- unum Sedimen...in loco dicto Satgoslò. 1546.4.12 (29)

- Sagoslò. 1558.31 .5 (31)

- in loco chiamato drio Goslò. 1609.7 .10 (33)

- in Curnich Sagoslò. 1667.12.10 (40)

- Affittanza Commun di San Giorgio del Monte Sagoslò, o sia drio Goslò. 1667.3.11 (42)

58 Tra il 1558 e il 1778 su 9 menzioni di questo toponimo (scritto con una o due $t$ ), una sola suona Segata, forma che ricompare però in un recente rilievo da Bila: ...un Segato cieua itit. / 'Cieua pociasu si vilest, / us uincie te Sagatine... (Ce ne andremo in Sagata./ Con calma supereremo / $\mathrm{i}$ tornanti di Segata - Di Lenardo $1974 n^{\circ} 40$ ). Zagata, completamente "di ragion" (proprietà) di quei di Bila, sarebbe un pendant di Njïvica, solo che si trova a $200 \mathrm{~m}$ più in basso e, non essendo attorniata da alte montagne, è meno selvaggia, più aperta e "domestica".

59 L'informatore (N) conosce a Gözd la località Ta-za Horï̌ico 'Dietro la Piazzetta', luogo dove, in altri tempi, si riunivano i bambini per giocare. 
- Sentenza a favor del Commun di S. Giorgio contro Danificatori nel Monte Satgoslò, o sia drio Goslò. 1668.12.5 (43)

- il Bosco drio Goslò, ovvero Curnich. 1668.23.5 (179)

- Sagata con suo Pascolo drio Goslò. 1680.28 .3 (44)

- in un loco drio Goslò ovvero Curnich. 1718.20 .10 (181)

- e drio Goslò. 1746.29.5 (49)

- con la somità del Monte drio Goslò fino alla Confin dei Stoluizani. 1749.3.3 (50)

- Trabnilidù drio Goslò. 1749.15.5 (51)

- Monte drio Goslò. 1750.12.10 (52)

- Siti detti dietro Goslò. 1756.5.5 (127)

- dietro Goslò. 1756.5.5 (128)

- drio Goslò. 1756.5 .5 (129)

- volgarmente detto drio Goslò. 1757.26.10 (139)

- luoghi detti drio Goslò. 1757.21.12 (140)

- Pascolo, e Bosco situato dietro Goslò. 1757.21.12 (141)

- dietro Goslò. 1757.27.2 (151)

- drio Goslò. 1758.8.6 (157), 1759.18.4 (153), 1777.25 .2 (214)

- un pezzo di Comugna con Bosco, e Selva situata nelle Pertinenze di San Giorgio in loco chiamato drio Goslò. 1778.12.3 (217)

- drio Goslò. 1779.22.6 (223, 225), 1780 (235)

- drio il Goslò. 1780 (236)

- drio Goslò. 1780.18.4 (244, 245)

- ...nelli soli siti Boschivi, e Pascolivi Compresi tra il Fiume Resia da una parte e li due Rivi Uragnipotoch, e Brusinipotoch, sino al Confine di Osseacco dall'altra, che comprendano anche una picciola parte delle varie situazioni abbraciate dalla generale denominazione di drio Goslò. 1780.18 .4 (246)

- drio Goslò. 1780.10.6 (250), 1782.7.2 (319), 1782.12.2 (322)

\section{4. *Za Hözdi: Ta-za Hözdi (N)}

- tutto lo Monte, comenzando zoso del Fiume Ucea per mezzo, \& a rimpetto lo loco detto da Piè del Miruhic, $\&$ andando suso per dritta Linea per sino drio Sagosdì, ove che è fatta una Croce in Sasso per sino alla summità di esso Monte, per donde sono signate in quatro Sassi quatro Croce, \& traversando per de drito verso Stoluizza sino per mezzo lo Rivo chiamato Lastiguipatoch (!), caminando drio due Croce signate in due Sassi. 1580.9.6 (14)

- indi a Masasnati Clin, indi suso alla sommità di Sagoldi [Sagosdi], ove si trova uno (!) Croce scolpita in un Sasso, indi calandosi per la Monte Sagoldi sino nel Rivo Ucea appresso li Casoni di San Giorgio, indi suso per il Mirnich... 1777.29.4 (212) 
- calandosi per la Monte Sagosdi fino al Rivo Ucea. 1779.22.6 (227)

- Sagosdi. 1780.10.6 (253)

\section{Zalëni Potök (O), Zelëni Patök (B) ${ }^{60}$}

- ...per il Livinale sino in Mecia, o sia Rivo Bianco, indi verso ponente in Pasigh, e seguitando per traverso sino Zelenipotoch. 1777.29.4 (212)

\section{Zapolúdnik, Ta-za Pulúdnik (B) ${ }^{61}$}

- nelli siti, e Territorio di Braman, e Sapoloduvich... 1721.21.11 (79)

- i Legnami tagliati nei siti promiscui di Sappolodvich, e Barman. 1722.24.1 (80)

- Sopoluduich. 1722.7.3 (82)

- Sappolodovich, o sia Sapolunich. 1722.22.3 (84)

- Sappolodovich; Sappolodvich. $1722.8 .10(86)$

- Cappolodovich (!); Seppolodovich. 1722.17 .10 (90)

- Sappulodonich. 1723.4.11 (93)

- Sappulodnich (94), Sappulodovich (95), Sappulodnich, Sappolodnich (96), Sappuludnich $(97,98,99) 1723.9 .11$

- Soppolodvich. 1724.1.4 (101, 102)

- Sopolodvich. 1724.27.4 (104)

- Sappalodnich. 1724.17.6 (196)

- Sapolodovich. 1725.13.1 (112), 1725.16.3 (113), 1725.7.5 (117)

- un suo pezzo di Medilli nel Monte Sapoludnich chiamato Jacumonepotoch. 1736.13.10 (339)

- Sapoludnich. 1736.13.10 (340)

- negando di bel nuovo, che i Siti di Sapolodnich, e Palunich siano lo stesso. 1736.1.12 (342)

- Sapulundenich. 1778.12.3(220)

- Sapolunich. 1780.10.6 (250)

- Sappolludinich. 1780.10.6 (254)

- Sapalovich. 1780.4.9 (265)

- Sappoludnich. 1781.13.2 (300) [correzione a stampa su foglietto già incollato sopra la dicitura Supolsinich]

60 Questo toponimo, rilevato nella zona di Karnica / Ucja, compare anche nei dintorni di Bila come Zelëni patök (B).

61 Nel nostro elenco alfabetico, questo toponimo compare in una ventina di forme, tutte del Settecento, alle quali però da altri documenti storici si potrebbero aggiungerne altre, più vecchie e curiose, come per es. un Postpolutnig, ibrido latino-resiano. Per quanto riguarda invece la presenza di Zapoludnik nella cronaca poetica della valle, si veda la nota 38 s.v. Mïzica. 
- Sapolodnich. 1781.6.3 (278)

- Sappaludnich; Sapoludnich. 1781.25.8 (353)

- Sapolunich. 1782.9.7 (311)

\section{Zorgnale}

- andando sino alla Sommità del Monte Musi, e per Apice da Cadin in Zorgnale, indi in Lavora, poi in Goliz giù per la Creta, fino drio Pustigast, fino a Bresaviza, \& indi alla Resia, e Niscipotoch. 1778.12.3.(219)

\section{Conclusioni}

4. Dopo aver presentato al pubblico scientifico questa discreta raccolta di toponimi resiani, è ora di accomiatarci. Per caratterizzare in breve il valore - almeno relativo del nostro elenco di nomi resiani, ci è venuta l'idea di ricorrere a un' analogia, traendola dalla numismatica. La nostra stampa per liti (fino al 1982 - anno della pubblicazione Madotto - nessuno ne sapeva nulla), dalla quale sono emersi parecchi bei nomi storici, non è forse paragonabile a un vaso di monete ritrovato nell'orto o in una casa diroccata? Vero è però che non ogni deposito di vecchie monete rappresenta già di per sè un tesoro favoloso, non tutti i conii sono in metallo prezioso, il loro stato di conservazione non è sempre buono, tra i singoli pezzi molti si ripetono, pochi - o nessuno - sono "pezzi unici", e via di seguito. Comunque, se gli studiosi di numismatica sanno apprezzare e valorizzare ogni sia pur modesta scoperta nel loro settore di attività, coscienti come sono che è già un discreto successo quando si possono individuare inattese e valide presenze, frequenze, curiose somiglianze e parentele, diffusione sicura in un dato spazio e/o tempo, perché dovrebbero essere da meno gli studiosi di toponomastica? I nostri toponimi storici, pur incongruenti nella scrittura, non alieni da banali errori di trascrizione o di stampa, limitati al periodo 1382-1784, circoscritti a due comuni della Val Resia (Bila e Njïwa), appaiono linguisticamente collegati gli uni con gli altri, come se si dessero la mano, proveniendo da località contigue, "ad invicem se tenentes".

4.1. Tuttavia non ce la sentiamo di finire senza rispondere a una domanda che sappiamo quanto mai viva, anche se non ci è stata rivolta direttamente. Cerchiamo pertanto di dare qui almeno qualche notizia sommaria sul decorso degli studi di toponomastica resiana. Naturalmente non possiamo entrare nei dettagli e citare pubblicazioni dove ricorre solo qualche singolo nome resiano, ma ci limiteremo a scritti di un certo rilievo.

4.1.1. Un lontano precursore ne è senz'altro il ceco Antonin Pišely, capitato nel 1801 per servizio nella valle di Resia o al suo margine occidentale, dove raccolse alcune dozzine di voci resiane. Quasi più che per via dei dati (modesti: 16 toponimi e non tutti precisi!) il suo contributo merita forse di essere ricordato per la distribuzione sistematica in nomi di villaggi, idronimi, oronimi e microtoponimi. ${ }^{62}$ 
4.1.2. Strano che - eccetto Pišely - fra quanti dalla fine del Settecento e a tutta la prima metà dell'Ottocento visitarono la valle sotto il Canin, lasciandoci descrizioni di vario genere, memorie o altre tracce scritte, ${ }^{63}$ nessuno abbia pensato di segnalarci nomi di luogo che escano dalla cornice convenzionale (nome del capoluogo e pochissimi altri che si possono contare sulle dita).

4.1.3. I rilievi cartografici che si svolgono su incarico dei Quartieri generali sono sempre segreti ed è pertanto logico che così sia stato anche col lavoro della commissione del Q. G. austriaco. Sempre ci rimane però a disposizione almeno il risultato definitivo, fissato sulle carte a piccola scala, con maggiore o minor numero di toponimi, più o meno fedeli a quanto dettato dagli informatori. Ciò che scrisse Joseph von Bergmann nel $1848^{64}$ e nel $1849^{65}$ non ci consente certo di vedere dietro le quinte del "teatro" geografico-militare austriaco. Per avere chiarimenti sui toponimi resiani e consigli sul modo di trascriverli, il Bergmann si era rivolto a un'autorità indiscussa: il linguista sloveno F. Miklošič ${ }^{66}$ allora bibliotecario nella Biblioteca di Corte viennese.

4.1.4. La seconda metà dell'Ottocento vide approdare nella Resia il famoso linguista polacco J. Baudouin de Courtenay, salpato però da un porto della Russia: Pietroburgo. Al suo primo soggiorno nella valle (agosto 1873) seguirono altre visite $\mathrm{e}$ soste; sicché, fino allo spirare del secolo, non si può pensare nulla di filologicamente importante che riguardi Resia senza l'apporto attivo del $\mathrm{BdC}$. Dal momento che la sua figura umana e scientifica domina su tutti e su tutto, non ci deve sorprendere che pure il contributo di tale linguista alla toponomastica resiana superi ciò che in questo campo specifico si è fatto sia prima che dopo di lui, quasi fino al giorno d'oggi. ${ }^{67}$

62 "Feldkurat", cappellano militare austriaco nel reggimento "K. Kinsky", che per hobby si dedicava alla raccolta folkloristica, con lettera del 14 aprile 1801 invio una colletta di 73 voci resiane a Praga, al suo amico J. Dobrowsky, il quale provvide a pubblicarle: "Über die Slawen im Thale Resia" in Slawin I,2, 1806, pp. 120-128; una nuova edizione, curata da W. Hanka, uscì nel 1834.

63 Oltre J. Potocki (data imprecisata: 1791-1797) sono da ricordare L. L. Linussio ("Solwizza, primo agosto 1821"), M. Tenore (Resiutta, 2 nov. 1824), G. Fresco (1840), I. I. Sreznevski (fine di aprile 1. maggio 1841), S. Vraz (intorno al 10 giugno 1841).

64 Bergmann $(1848,46-50)$ scrive tra l'altro che pastori e legnaioli di lingua slava, stabilitisi in questa valle (Resia) non si sa quando, denominarono valli e monti, terreni, boschi, ecc. Adduce parecchi toponimi, traendoli dalla sezione del Q. G. austriaco ed apponendovi l'interpretazione del Miklošič.

65 Allo scritto del Bergmann (Das slavische Resiathal, 1849, pp. 29-32) era allegata una cartina geografica della valle.

66 Il contributo del Miklošič alla toponomastica resiana è stato di recente esaminato da H. Steenwijk 1992, cap. 4: Miklosič und resianische Toponyme.

67 Molto, se non tutto il materiale toponimico raccolto dal BdC nel 1873 è sparso nei suoi Materiali I, 1895, ma rintracciabile senza difficoltà tramite il ricco indice alle pp. 671-2; riunito insieme sta all'inizio della monografia "Rez'ja i Rez'jane" del 1876; disposto in ordine alfabetico si troverà come capitolo a sè nel Vocabolario resiano (Rezijanskij slovar) del BdC, che stanno preparando per la.stampa A. Duličenko, M. Matičetov e N. I. Tolstoj. Nuovi toponimi resiani, inediti, raccolti sempre dallo stesso autore, si trovano nell'archivio dell'Accademia di scienze russa, sez. di Pietroburgo (in copia presso l'Istituto di tradizioni popolari slovene della SAZU a Lubiana). 
4.1.5. Per recuperare, cioè tirare fuori dalle nebbie medievali certi nomi storici, ci volevano metodi e conoscenze particolari (specialmente in materia di paleografia), che solamente la storiografia può metterci a disposizione: Fu così che prestarono la loro opera - poco meno che di scavo: da parte friulana A. Di Prampero, V. Joppi, da parte slovena F. Kos, da parte tedesca A. Jaksch e altri che qui non è il caso di citare. Non è lecito però sottacere un entusiasta di storia dell'alpinismo, A. Gstirner (1906), ${ }^{68}$ il quale, risalendo indietro fino dove era possibile, ha saputo trarre da ogni dove nomi storici e poi, avvalendosi della sua singolare domestichezza con le montagne tutt' intorno a Resia, anche localizzarli con precisione, aggiudicandoli ai loro primi veri o probabili portatori (si pensi per es. a un "Mons Habilis" o altri nomi simili, più o meno refrattari all'identificazione).

4.1.6. Tra gli innamorati della nostra montagna è doveroso ricordare altri due personaggi: J. Kugy e H. Tuma. Al primo ritorneremo ancora, poiché tra le sue visioni poetiche e altamente umane ci fornisce un meraviglioso passo a commento e chiusura di questo nostro contributo. Del Tuma invece, alpinista e insieme raccoglitore instancabile dei nomi di montagne (il suo scritto forse più noto è dedicato alla "Toponomastica delle Alpi Giulie" 1929), senza voler diminuire alcun merito - e ne ha tanti! - penso che, almeno nel settore resiano, sia sempre valido quel che sta scritto come corollario ai n.ri 5 e 13 di Resia. Bibliografia ragionata (1981).

4.1.7. I nomi di luogo resiani devono aver destato l'attenzione anche di Bruno Guyon, uno slavista originario della Val Natisone (Barnas/Vernasso 1868 - Napoli 1943); intorno al 1933-34 egli avrebbe scritto per la "Zeitschrift für Ortsnamenforschung" alcuni articoli (fra l'altro: Die mediterranischen toponomastischen Elemente in Resiathal e Die slovenische Echtheit der Resianer aus den Ortsnamen in Resiathal), ${ }^{69}$ che però nel suddetto periodico non si trovano. ${ }^{70}$

4.1.8. Durante il seminario per la lingua, letteratura e cultura slovena che ogni anno si svolge presso l'Università di Lubiana; nel luglio 1972 fu organizzata un'escursione nella Val Resia. Vi prese parte anche il prof. Kronsteiner che, entusiasta dell'incontro con la Valle e coi suoi abitanti, decise di ritornarci per conto proprio. Frutto tangibile della sua seconda visita è lo scritto Die Toponymie des Resia-Tales del 1975. Egli tentò un esperimento: prendendo per base il patrimonio onomastico resiano segnato sulla carta (CI) dell'Istituto Geografico Militare 1:25.000, si prefisse di controllarne la situazione 60 anni dopo i rilievi fatti nel 1910. Tuttavia, la ristretta scelta degli informatori (provenienti, pare, soprattutto dal villaggio più grande e popolato Oso(j)ane) ha probabilmente limitato i risultati della ricerca sul campo. Solo così è

68 Particolarmente importante il capitolo B: Der Mensch in den Bergen.

69 Notizie tolte dall' articolo di Jem, Bruno Guyon, in Primorski SBL, 6, Gorica, GMD, 1979.

70 Da gent. comunicazione del prof. O. Kronsteiner. Sarebbe interessante appurare quale fine abbiano fatto questi ed altri scritti del Guyon, se stampati altrove o rimasti inediti presso gli eredi o depositati in qualche biblioteca. $\mathrm{Ci}$ raccomandiamo per questo alla cortesia degli slavisti dell'Istituto Universitario Orientale di Napoli. 
comprensibile la qualifica "ignoto" (unbekannt) a fianco dei nomi che invece si potrebbero benissimo dire "arcinoti": così per esempio (restando nei limiti delle prime lettere dell'alfabeto) BRUSSEN PATOK (v. la nostra nota 21), CELZE (v. le note 9 e 31), CLADIE, COCHIAZE (v. nota 38). Per altri nomi credo che non sia stata considerata l'eventualità che ci troviamo di fronte a evidenti sbagli di stampa: per es. ANARCOTA (Auarcota o Warkòta, quindi nessun rapporto col frl. cort "Hof, Bauernhof" che in resiano si chiama dwör); per GOSTO la forma corretta è Goslö e al posto di DRIO GOSTO dobbiamo vedere un SATGOSLO (1546), *Zagoslö, nel 1993 registrato come Ta-za Goslòn (B; si veda pure la nostra nota 26). Infine il nome IEDAIE "Felsgruppe" (anch'esso "ignoto"!) fu inserito in una poesia moderna: tuw iseh jedajeh / punčikeh paci (R. Quaglia 1985, 26), che alla lettera dovrebbe suonare: in queste Jedaje / piene di pietre, mentre il poeta diede a jedaje un senso metaforico di "luogo solitario" e di conseguenza il traduttore scrisse: "v tej samoti / polni kamenja."

4.1.9. Ed ecco infine giunto il momento di ritornare al Kugy, poeta sensibilissimo, il quale delle sue montagne predilette - del Triglav per esempio - non esitò un istante a dire "che cresceva davanti ai miei occhi fino ad assumere una sua personalità precisa e potente, che $\mathrm{m}$ 'incatenava tanto più, quanto più a fondo ero riuscito a penetrare nella sua essenza e sviscerare il suo significato". Questi suoi nobili e profondi sentimenti egli era persuaso di poterli condividere con i datori (o "creatori") dei nomi, come egli si esprime, insomma con l'anima del popolo. Se egli ebbe sott'occhio in primo luogo i nomi delle montagne, ciò non significa che non si possa estendere il suo ragionamento dagli oronimi agli idronimi $e$ in genere a tutti $i$ toponimi, compresi perfino $i$ microtoponimi.

Ed ora riproduciamo, Dalla vita di un alpinista, quanto il nostro corregionale Julius Kugy scrisse nel 1917 - come apposta per noi - e pubblicò per la prima volta a Trieste settanta anni fa (Aus dem Leben eines Bergsteigers):

«Direi che anche l'anima popolare senta così e batta queste vie, creando i nomi dei suoi monti dopo averne afferrati con precisione il carattere e l'individualità attraverso l'osservazione secolare. Per questo bisogna trattare con rispetto e con amore i nomi autoctoni e popolari, bisogna ricercarli dove sono caduti nell'oblio e vegliare gelosamente perché non vengano mutati a capriccio o sostituiti con altri, di maniera. Col loro suono caratteristico e nella loro crudezza originale sono diventati una parte dell'individualità del monte, e spesso sanno ridare, meglio di qualunque descrizione $\mathrm{e}$ con vera poesia, l'impressione, per cui i monti uscirono dalla notte dei tempi ed entrarono nel raggio di osservazione e nella coscienza del popolo.» (Versione di E. Pocar, Trieste, $1985_{3}$, p. 55).

Si specifica che nel presente lavoro i capitoli 1. e 4., le note n. 1-7, 9-13, 19-23, 25-27, 29-36, 38, 39, 41-43, 46, 50, 54-56, 61-70 sono opera di M. Matičetov; il cap. 2., le note $n$. 8, 14-18, 24, 28, 37, 40, 44, 47-49, 51-53, 59, 60 e i Riferimenti bibliografici sono da attribuire a R. Dapit; il cap. 3. e le note n. 45, 46 e 57 sono di entrambi gli autori che insieme hanno cercato di risolvere i vari problemi nati durante l'esecuzione del lavoro in comune, attraverso il reciproco scambio di informazioni ed esperienze. 


\section{Riferimenti bibliografici}

BAUDOUIN DE COURTENAY J. - 1876, "Rez'ja i Rez'jane", in Slavjanskij Sbornik, III, 1, Sankt-Peterburg.

- 1895, Materialy dlja južnoslovjanskoj dialektologii i etnografii. I. Rez'janskie teksty, Sanktpeterburg.

BERGMANN J. - 1848, "Das Thal Resia und die Resianer in Friaul", in Anzeige-Blatt für Wissenschaft und Kunst, 121 (Anhang zu Jahrbücher der Literatur 121), Wien, pp. 46-50.

- 1849, "Das slavische Resia-Thal", in Archiv für Kunde österreichischer Geschichtsquellen, II, 1-2, Wien, Kaiserliche Akademie der Wissenschaften, pp. 253-256.

DI LENARDO D. - 1974, Te rosaiansche uiśize. Canti resiani, Udine, SFF.

GSTIRNER A. - 1906, "Die Julischen Alpen", in Zeitschrift des Deutschen und Oesterreichischen Alpenverein, 37, München.

KRONSTEINER O. - 1975, "Die Toponymie des Resia-Tales", in Wiener Slavistisches Jahrbuch, 21, Wien, pp. 117-130.

KUMER Z. - 1975, Pesem slovenske dežele, Maribor, Obzorja.

MADOTTO A. - 1982, La Val Resia ed i suoi abitanti, Udine-Mariano del Friuli.

- 1987, Vivere fra le montagne, Udine, Designgraf.

MATIČETOV M. - 1965-67, "«Canzoni resiane». Un foglio volante stampato a Gemona nel 1930", in Ce fastu?, 41-43, Udine, pp. 453-459.

- 1968-71, "Contributi allo studio del tema narrativo »corsa per il confine«.", in Ce fastu?, 44-47, Udine, pp. 53-57.

- 1972, Rožice iz Rezije, Koper-Trst-Ljubljana, Lipa-ZTT-ISN SAZU.

- 1973, Zverinice iz Rezije, Ljubljana-Trst, Mladinska Knjiga-ZTT.

- 1974-75, "Vecchie e nuove fiabe d'animali dalla Val Resia", in Ce fastu?, 50-51, Udine, pp. 110-118.

- 1978, "Ob zibki ljudske lirične pesmi v Reziji", in Govor, jezik in besedno ustvarjanje $v$ Beneški Sloveniji, Špeter Slovenov-Trst, Študijski center Nediža-ZTT, pp. 57-80.

- 1981, Resia. Bibliografia ragionata, Udine, Graphart.

- 1982, "Sulla »Lingua Illirica, o sia Reseana«", in Metodi e Ricerche, 1, n. 2, Udine, pp. 94-95.

- 1992, "Resia. I. Dimensione linguistica", in La cultura popolare in Friuli "Lo sguardo da fuori" (Atti del convegno di studio, G. Fornasir e G.P. Gri, a cura di), Udine, Accademia di Scienze Lettere ed Arti, pp. 57-94

MERKÙ P. - 1976, Ljudsko izročilo Slovencev v Italiji. Zbrano v letih 1965-1974 / Le tradizioni popolari degli sloveni in Italia. Raccolte negli anni 1965-1974, Trst/Trieste.

QUAGLIA R. - 1985, Baside, Trst, ZTT.

Resia. Numero unico. Primavera 1967, Udine, SFF. 
SIMONETTI A. - 1993, L'antica tecnica della fluitazione del legname mediante l'utilizzo di chiuse. Le "stùis di Tralbe" a Moggio Udinese, Pontebba (Udine), Comunità Montana Canal del Ferro-Val Canale.

STEENWIJK H. - 1992, "Miklošič als Resianologe", in Miklošičev zbornik. Mednarodni simpozij v Ljubljani od 26. do 28. Junija 1991, Ljubljana, SAZU-Univerza v Ljubljani (FF)-Univerza v Mariboru (Obdobja 13), pp. 451-461. TUMA H. - 1929, Imenoslovje Julijskih Alp, Ljubljana, Slovensko Planinsko Društvo.

\author{
Povzetek \\ REZIJANSKA KRAJEVNA IMENA \\ $\mathrm{v}$ tiskanem pravdnem zborniku izpod konca 18 . stoletja
}

\begin{abstract}
Ob pomembnih pravdah, posebe medobčinskih, ki so se včasih vlekle v neskončnost, medtem pa so se akti tako namnožili, da se je bilo med njimi skoraj nemogoče znajti, so prizadeti (sami od sebe ali na zahtevo sodišč?) dali celotno dokumentacijo razmnožiti $v$ tiskarni, da bi sodniki, zagovorniki in drugi sodni udeleženci $z$ obeh strani, imeli razpravno gradivo pri rokah v pregledni obliki. Dobro ohranjen, na 388 straneh $\left(4^{\circ}\right.$, s.l., s.a., vendar taqn. 1784) tiskan izvod takega zbornika listin iz pravde med rezijanskima občinama Bila (S. Giorgio) in Njiva (Gniva) je v svoji knjigi La Val Resia ed i suoi abitanti iz leta 1982 prvič omenil njegov hranitelj, rajni Aldo Madotto. Le-ta je istočasno posodil svoj mikavni zbornik M. Matičetovemu, da si je iz njega izpisal - ad notam et litteram - vsa imena krajev v rezijanski dolini. Kartotečni listki s tistimi imeni so potlej obležali v predalu do letos, ko je furlanski lingvist R. Dapit (ki zbira in obdeluje toponomastiko Rezije za tamkajšnjo občinsko upravo), z veseljem sprejel soavtorjevo ponudbo, ne samo da prenese s starinsko patino prekrita imena iz let 1382-1784 na računalnik, ampak da hkrati tudi preišče, na terenu, koliko jih še živi in kako se glasijo dandanes, ko je od takrat preteklo od šeststo do dvesto let.

V uvodnih dveh poglavjih sta avtorja prispevala - vsak zase - najnujnejše informacije o svojem del(ež)u, pa še nekaj spoznanj in priložnostnih misli o teh zgodovinskih zapiskih in njih pomenu za rezijanologijo. Središčni, poglavitni del te objave pa je 3. poglavje, sad skupnega dela, ki prinaša imena, razvrščena abecedno $v$ izvirni podobi ( $z$ napakami vred, s ponavljanji, z nekaj konteksti, kjer so navedeni prostorsko med sabo povezani kraji "Montes contiguos et ad invicem se tenentes" ipd.), zraven pa še imena, kakor jih poznajo domačini v našem času.

Na koncu je dodan še sklep (pogl. 4), ki naj nakaže - vsaj povrhu - kdo in kako je v zadnjih dveh stolettjih kaj prispeval k poznavanju rezijanskih krajevnih imen.

Marsikaj poučnega in branja vrednega pa sta pisca vtaknila v sedemdeset opomb, ki dopolnjujejo védenje o imenih tudi s podatki, strogo vzeto niti ne čisto jezikoslovne narave.

O svojem "hiševanju" sta dolžna dati še tale nadrobni pregled: M. Matičetov je prispeval uvodno poglavje 1 in sklepno pogl. 4, zraven pa še opombe št. 1-7, 9-13, 19-23, 25-27, 29-36, 38, 39, 41-43, 46, 50, 54-56, 58, 61-70; R. Dapit je prispeval uvodno poglavje 2, opombe št. 8, 14-18, 24, 28, 37, 40,44,47-49,51-53, 59, 60 in bibliografski pregled. Opombe št. 45,46 in 57 so skupne. Pisca sta kajpada skupaj in sproti reševala vprašanja, ki so nastajala pri delu in se medsebojno opozarjala na gradivo, ki bi lahko obogatilo skupni prispevek.
\end{abstract}

